\title{
Logarithmic conformation reformulation in viscoelastic flow problems approximated by a VMS-type stabilized finite element formulation
}

\author{
Laura Moreno ${ }^{1}$, Ramon Codina ${ }^{1,2}$, Joan Baiges ${ }^{1}$ and Ernesto Castillo ${ }^{3}$ \\ ${ }^{1}$ Universitat Politècnica de Catalunya, Jordi Girona 1-3, Edifici C1, 08034, Barcelona, Spain \\ ${ }^{2}$ Centre Internacional de Mètodes Numèrics en Enginyeria, Gran Capità S/N, 08034 Barcelona, Spain \\ ${ }^{3}$ Universidad de Santiago de Chile, Av Libertador Bernardo O'Higgins 3363, Santiago, Chile
}

\begin{abstract}
The log-conformation reformulation, originally proposed by Fattal and Kupferman [1], allows computing incompressible viscoelastic problems with high Weissenberg numbers which are impossible to solve with the typical three-field formulation. By following this approach, in this work we develop a new stabilized finite element formulation based on the logarithmic reformulation using the Variational Multiscale (VMS) method as stabilization technique, together with a modified log-conformation formulation. Our approach follows the term-by-term stabilization proposed by Castillo and Codina [2] for the standard formulation, which is more effective when there are stress singularities. The formulation can be used when the relaxation parameter is set to zero, and permits a direct steady numerical resolution. The formulation is validated in the classical benchmark flow past a cylinder and in the well-known planar contraction 4:1, achieving very accurate, stable and mesh independent results for highly elastic fluids.
\end{abstract}

Keywords: Viscoelastic fluids, Log-conformation, Stabilized finite element methods, Multiscale, Contraction 4:1, Flow around a cylinder.

\section{Introduction}

Viscoelastic fluids are widely employed in a large variety of engineering, medical and natural science applications, such as safety devices capable of absorbing impacts, some plastics or blood pumps. Moreovear, these fluids display distinct features to those shown by pure viscous fluids, for instance turbulent drag reduction [3] or elastic turbulence at low Reynolds number [4], among others. For these reasons, the study of numerical tools for the computation of viscoelastic flows is valuable both from the fundamental and the practical perspectives. The main characteristic about the behavior of viscoelastic materials is that stresses do not just depend on current deformation rates, but also on the deformation history. Due to this, time is a crucial element to take into account, because viscoelastic fluids have different responses depending on the time-scales of the deformation.

The computation of the flow of these kind of fluids leads to several difficulties, above all when elasticity becomes dominant, i.e. the dimensionless number known as Weissenberg number is high. This number is defined by $\mathrm{We}=\lambda u / L$, where $\lambda$ is the characteristic relaxation time of the material $u$ is the characteristic velocity of the flow, and $L$ is the characteristic length of the domain. In some articles, another dimensionless number is used for flows with non-constant deformation rate, the Deborah number, De $=\lambda / t_{c}$ written in terms of the relaxation time and the time-scale of observation $t_{c}$. In [5] an extensive discussion is done about the suitable uses of each number. 
In addition, the difficulties in numerically simulating high Weissenberg numbers flows are one of the biggest challenges in computational rheology since the 1970s; it is called the High Weissenberg Number Problem (HWNP) [6]. It is defined as a numerical phenomenon that causes the iterative computations to breakdown for relatively low Weissenberg numbers, and normally it is expressed as a lack of convergence in the iterative method due to the hyperbolic nature of the differential constitutive equations. The breakdown occurs for a critical value of the Weissenberg number, but it is specific to each particular problem, the spatial discretization and the numerical algorithm. The numerical instability is brought about by the failure of the proper balance of the deformation rate and the convection, and it was identified and discussed by Fattal and Kupferman [7]. It is a fundamental instability, present in all constitutive models and standard numerical methods. Nevertheless, it is demonstrated that constitutive methods can predict other instabilities of mathematical character $[8,9]$, referred to as constitutive instabilities, which can be classified in two: the Hadamard instability, associated with the non-linear fast response of constitutive equations, and the dissipative instability, related to the formulation of the dissipative behavior of viscoelastic models.

The source of the HWNP has thus been identified: on the one hand the loss of positivedefiniteness of the conformation tensor, an internal variable which should be symmetric positive-definite to be physically admissible $[10,1]$; on the other hand, the large stress gradients, regions with particular high deformation rate, or near stagnation points favor the breakdown of the numerical method, as explained in Fattal and Kupferman in $[1,7]$. They describe the cause for this phenomena to be caused by the use of inapropriate approximations to represent the stress tensor, remarking the importance of preserving its positivity. By following these ideas, a new formulation was proposed by Fattal and Kupferman [1]: the log-conformation representation (denoted by LCR), a reformulation of the traditional equations of viscoelastic fluids, which eliminates the instability and linearizes the exponential stress profiles near the stress singularities. Therefore, the formulation seeks to treat the exponential growth of the elastic stresses, allowing to extend the range of Weissenberg numbers for which a numerical solution can be obtained.

However, alternative schemes have been proposed in other works. For example, Vaithianathan and Collins [11] presented two matrix decomposition schemes in order to construct the positive definite conformation tensor, employing the FENE-P model. Balci et al. [12] proposed a square root conformation representation. Afonso et al. [13], developed several matrix kernel-transformation families which can be applied to the conformation tensor equation. Nevertheless, although there are a variety of proposals to deal with the lack of positive-definiteness in the conformation tensor, the logarithm representation is the uniquely capable of linearizing the exponential stress profile.

Since the logarithmic formulation was presented, a great number of works have been written following this novel strategy, applying different methodologies and schemes, in finite volume and finite element (FE) codes. The next first work published applying the log-conformation reformulation is due to Hulsen et al. [14] using the DEVSS/DG formulation for the discretization and a first-order upwind scheme in a FE implementation. Later, Coronado et al. [15] proposed a "simple alternate" form of the log-conformation formulation implemented in the DEVSS-TG/SUPG FE method, and in comparison with the previous work, fewer code modifications with respect to the standard formulation were required. An analysis between the two previous publications and two new implementations was presented by Kane et al. [16], remarking particularly the treatment of the advective term of the constitutive equation. The final conclusion is that all four formulations are very similar, except the one described by Coronado et al. [15], that is a little less robust due to the linear interpolation of the convective term. Damanik et al. [17] defined a fully coupled monolithic FE approach, using the edge-oriented FE stabilization for the 
convective term. Saramito [18] and Knechtges [19] recently derived fully implicit versions of the log-conformation formulation that do not involve an algebraic decomposition of the velocity gradient tensor, and which can be linearized and solved by the Newton-Raphson method. Afonso et al. [20, 21] investigated the performance of the log-conformation reformulation using the finite volume method framework in both works, although the second is more interesting because it seeks to predict the rich dynamical transitions in the $4: 1$ contraction planar benchmark, whereas the first one solves the flow around a cylinder, a problem without singularities. Comminal et al. [22, 23], simulated incompressible viscoelastic flows and the stream-log-conformation methodology, a combination between the log-conformation with the stream function flow formulation (see [24]) that is beneficial for the accuracy and stability of the numerical algorithm. One of the most recent publications belongs to Pimenta et al. [25], who increased the robustness and accuracy of the viscoelastic solver in OpenFoam, implementing there the log-conformation methodology.

Concerning this work, we propose a slightly different formulation to the log-conformation formulation. Our formulation is non-singular when the Weissenberg number is close to zero, while the original one proposed by Fattal and Kupferman [1] presents some problems because of the inverse of the relaxation time in the equations. The same idea was followed by Saramito [18]; both formulations can be reduced to the Navier-Stokes equations when the Weissenberg number is set to zero. Due to this, continuation methods can be successfully employed to get the optimal convergence in the validated problems. Also, we have to remark that the steady problem can be solved directly, while in most of the references the logarithmic formulation shows a strong time-dependency. As Saramito [18] pointed out, the reason can be that previous methods relied on some finite difference methods for computing the Jacobian matrix and strong non-linearities were considered non-differentiable (see, for example, [17]). Let us stress again the theoretical contribution presented in [18], where the main properties of the typical operators of the formulation are proved.

In this paper, we apply the efficient logarithmic reformulation to solve the viscoelastic problem with the goal of simulating flows with a high Weissenberg number, using a stabilized formulation based on the Variational Multiscale (VMS) method. This stabilized formulation has its beginnings in the methods introduced by Hughes et al. [26] for the scalar convection-diffusion-reaction problem, and later extended to the Navier-Stokes problem by Codina $[27,28,29]$, where the space of the sub-grid scales was taken as orthogonal to the FE space. This idea was adapted to the three-field Navier-Stokes problem in [30] and later to the viscoelastic flow problem in [2]. The mathematical analysis of the formulation can be found in $[31,32]$. Other papers can be found concerning the VMS framework for viscoelastic regimes, such as $[33,34]$, where an Oldroyd-B fluid is considered. By following the same steps, the purpose of the present paper is the design of stabilized formulations which allow computing viscoelastic problems with a high Weissenberg number using the logarithmic reformulation, and testing them for numerical examples where both elastic stress gradients and numerical singularities are the main features.

This work is organized as follows: Section 2 explains in detail the log-conformation reformulation and the modifications considered; later we report the steps to obtain the strong formulation, the variational equations and consequently the Galerkin FE discretization. Once the main equations are defined, Section 3 presents the stabilized FE approach based on the VMS approach; the linearization of the problem is also extensively discussed.

As for the numerical results, they are presented in Section 4. First, in Subsection 4.1, a study of the $h$ convergence of the formulations is described for a stationary OldroydB flow, where a manufactured solution is considered. Secondly, in Subsection 4.2, the flow past a cylinder is tested for an Oldroyd-B fluid, comparing the solution obtained for Weissenberg numbers $0.6,0.7$ and 0.9 with other published solutions. The drag force on 
the cylinder is also contrasted for higher Weissenberg numbers. Then, the well-known 4:1 planar contraction benchmark is elaborated in Subsection 4.3. It is studied for two different Reynolds number values. Finally, we present a three dimensional example in Subsection 4.4, with the aim of showing that the formulation works well in 3D cases. Conclusions are drawn in the last section of the paper, Section 5.

\section{The modified log-conformation formulation problem}

\subsection{Standard formulation for the viscoelastic flow problem}

Let us start presenting the standard equations associated to the viscoelastic flow problem. Let us consider a viscoelastic fluid moving in a domain $\Omega$ of $\mathbb{R}^{d}(d=2$ or 3 ) during the time interval $\left[0, t_{\mathrm{f}}\right]$ and let $\partial \Omega$ be the boundary. Assuming the flow to be incompressible and isothermal, the governing equations are the conservation of momentum and mass, which can be expressed as follows:

$$
\begin{array}{r}
\rho \frac{\partial \boldsymbol{u}}{\partial t}+\rho \boldsymbol{u} \cdot \nabla \boldsymbol{u}-\nabla \cdot \boldsymbol{T}+\nabla p=\boldsymbol{f} \text { in } \Omega, t \in\left(0, t_{\mathrm{f}}\right), \\
\nabla \cdot \boldsymbol{u}=0 \text { in } \Omega, t \in\left(0, t_{\mathrm{f}}\right),
\end{array}
$$

where $\rho$ denotes the constant density, $p: \Omega \times\left(0, t_{\mathrm{f}}\right) \rightarrow \mathbb{R}$ is the pressure field, $\boldsymbol{u}: \Omega \times$ $\left(0, t_{\mathrm{f}}\right) \rightarrow \mathbb{R}^{d}$ is the velocity field, $\boldsymbol{f}: \Omega \times\left(0, t_{\mathrm{f}}\right) \rightarrow \mathbb{R}^{d}$ is the force field and $\boldsymbol{T}: \Omega \times\left(0, t_{\mathrm{f}}\right) \rightarrow$ $\mathbb{R}^{d} \otimes \mathbb{R}^{d}$ is the deviatoric extra stress tensor. In general, $\boldsymbol{T}$ is defined in terms of a viscous and a viscoelastic contribution as

$$
\boldsymbol{T}=2 \eta_{e} \nabla^{s} \boldsymbol{u}+\boldsymbol{\sigma},
$$

where $\eta_{e}$ represents the effective viscosity (or solvent viscosity), $\nabla^{s} \boldsymbol{u}$ is the symmetrical part of the velocity gradient and $\boldsymbol{\sigma}$ is the viscoelastic or the elastic stress tensor.

Finally, the constitutive equation for the viscoelastic stress tensor must be defined to close the problem. Even if there is a wide range of different models, we consider the Oldroyd-B model in this work, which is the model of a Newtonian stress supplemented with an extra-stress that satisfies the upper-convected Maxwell equation, and it reads as

$$
\frac{1}{2 \eta_{p}} \boldsymbol{\sigma}-\nabla^{s} \boldsymbol{u}+\frac{\lambda}{2 \eta_{p}}\left(\frac{\partial \boldsymbol{\sigma}}{\partial t}+\boldsymbol{u} \cdot \nabla \boldsymbol{\sigma}-\boldsymbol{\sigma} \cdot \nabla \boldsymbol{u}-(\nabla \boldsymbol{u})^{T} \cdot \boldsymbol{\sigma}\right)=\mathbf{0}, \text { in } \Omega, t \in\left(0, t_{\mathrm{f}}\right),
$$

where $\lambda$ is the relaxation time and $\eta_{p}$ represents the polymeric viscosity. Each term of the equation has a particular meaning: $\nabla^{s} \boldsymbol{u}$ is the source, $\frac{\lambda}{2 \eta_{p}} \boldsymbol{u} \cdot \nabla \boldsymbol{\sigma}$ represents the convective term and $\frac{\lambda}{2 \eta_{p}}\left(\boldsymbol{\sigma} \cdot \nabla \boldsymbol{u}+\left(\nabla \boldsymbol{u}^{T}\right) \cdot \boldsymbol{\sigma}\right)$ are the rotational terms. Note that from this point we write the polymeric and the effective viscosity in function of the total viscosity $\eta_{0}$. For that, an additional parameter $\beta \in[0,1]$ is introduced, so that $\eta_{e}=\beta \eta_{0}$ and $\eta_{p}=(1-\beta) \eta_{0}$.

Calling $\boldsymbol{U}=[\boldsymbol{u}, p, \boldsymbol{\sigma}], \boldsymbol{F}=[\boldsymbol{f}, 0, \mathbf{0}]$ and defining

$$
\begin{gathered}
\mathcal{D}_{t}(\boldsymbol{U}):=\left(\begin{array}{c}
\rho \frac{\partial \boldsymbol{u}}{\partial t} \\
0 \\
\frac{\lambda}{2 \eta_{p}} \frac{\partial \boldsymbol{\sigma}}{\partial t}
\end{array}\right), \\
\mathcal{L}_{0}(\hat{\boldsymbol{u}} ; \boldsymbol{U}):=\left(\begin{array}{c}
-\nabla \cdot \boldsymbol{\sigma}-2 \eta_{e} \nabla \cdot\left(\nabla^{s} \boldsymbol{u}\right)+\rho \hat{\boldsymbol{u}} \cdot \nabla \boldsymbol{u}+\nabla p \\
\nabla \cdot \boldsymbol{u} \\
\frac{1}{2 \eta_{p}} \boldsymbol{\sigma}-\nabla^{s} \boldsymbol{u}+\frac{\lambda}{2 \eta_{p}}\left(\hat{\boldsymbol{u}} \cdot \nabla \boldsymbol{\sigma}-\boldsymbol{\sigma} \cdot \nabla \hat{\boldsymbol{u}}-(\nabla \hat{\boldsymbol{u}})^{T} \cdot \boldsymbol{\sigma}\right)
\end{array}\right),
\end{gathered}
$$

we may write (2.1), (2.2) and (2.3) as

$$
\mathcal{D}_{t}(\boldsymbol{U})+\mathcal{L}_{0}(\boldsymbol{u} ; \boldsymbol{U})=\boldsymbol{F} .
$$




\subsection{The log-conformation reformulation}

Departing from the standard formulation, the logarithmic reformulation will be exposed, and later applied to the standard equations. This model has an interpretation in terms of statistical mechanics, which involves a statistical average of dyadic vector products. First, the conformation tensor is defined, taking into account that it must, by definition, be symmetric and positive-definite to be physically-admissible, because this internal variable represents the macromolecular configuration of the polymer chains. It is defined as

$$
\boldsymbol{\tau}=\frac{\lambda \boldsymbol{\sigma}}{\eta_{p}}+\boldsymbol{I}
$$

Consequently, the stress tensor can be expressed as a function of the conformation tensor as $\boldsymbol{\sigma}=\frac{\eta_{p}}{\lambda}(\boldsymbol{\tau}-\boldsymbol{I})$. Then, replacing $\boldsymbol{\sigma}$ in the constitutive equation (2.3) with $\boldsymbol{\tau}$, we can rewrite the Oldroyd-B model in terms of the conformation tensor $\tau$ as

$$
\frac{1}{2 \lambda}(\boldsymbol{\tau}-\boldsymbol{I})-\nabla^{s} \boldsymbol{u}+\frac{1}{2}\left(\frac{\partial \boldsymbol{\tau}}{\partial t}+\boldsymbol{u} \cdot \nabla \boldsymbol{\tau}-\boldsymbol{\tau} \cdot \nabla \boldsymbol{u}-(\nabla \boldsymbol{u})^{T} \cdot \boldsymbol{\tau}\right)=\mathbf{0} .
$$

However, in the paper, we have considered a modification when the conformation tensor is defined, with the aim of allowing $\lambda=0$, i.e., the Newtonian behavior. To this end, we introduce the relaxation-time parameter $\lambda_{0}(\lambda)$ linearly dependent with $\lambda$, which could be defined as $\lambda_{0}=\max \left\{k \lambda, \lambda_{0, \min }\right\}, k$ being a constant and $\lambda_{0, \min }$ a given threshold. So, if $k=1$ and $\lambda_{0, \min }=0$, the original change of variables proposed by Fattal and Kupferman [1] is recovered; however, if $k$ is taken equal to zero, then the three-field Navier-Stokes problem for Newtonian fluids is obtained. It is worth to remark that in the numerical experiments we have found useful to take $k$ small, so that $\lambda_{0}<\lambda$; this has allowed us to obtain converged solutions that we have not been able to get for $k=1$.

Thus, we define

$$
\boldsymbol{\tau}=\frac{\lambda_{0}(\lambda) \boldsymbol{\sigma}}{\eta_{p}}+\boldsymbol{I}
$$

From this point we use $\lambda_{0}$ instead of $\lambda_{0}(\lambda)$ to simplify the notation. The constitutive equation (2.3) can be rewritten by following the proposed modification as

$$
\frac{1}{2 \lambda_{0}}(\boldsymbol{\tau}-\boldsymbol{I})-\nabla^{s} \boldsymbol{u}+\frac{\lambda}{2 \lambda_{0}}\left(\frac{\partial \boldsymbol{\tau}}{\partial t}+\boldsymbol{u} \cdot \nabla \boldsymbol{\tau}-\boldsymbol{\tau} \cdot \nabla \boldsymbol{u}-(\nabla \boldsymbol{u})^{T} \cdot \boldsymbol{\tau}+2 \nabla^{s} \boldsymbol{u}\right)=\mathbf{0}
$$

The log-conformation reformulation basically consists of a change of variables in terms of the matrix-logarithm of the conformation tensor, that is to say, the conformation tensor is replaced by a new variable $\boldsymbol{\psi}=\log (\boldsymbol{\tau})$. This can be calculated through eigenvalue computation that rotates the $\boldsymbol{\tau}$ tensor into its main principle axes and can be expressed as $\boldsymbol{\psi}=\boldsymbol{R} \log (\boldsymbol{\Lambda}) \boldsymbol{R}^{T}$ because $\boldsymbol{\tau}$ is a symmetric positive definite tensor (for $k \leq 1$ ) and therefore it can always be diagonalized. In the expression introduced, $\boldsymbol{\Lambda}$ is a diagonal matrix with the eigenvalues of $\boldsymbol{\tau}$, and $\boldsymbol{R}$ is the orthogonal matrix of the eigenvectors of $\boldsymbol{\tau}$.

To sum up, in order to obtain the new formulation the stress tensor must be replaced by $\boldsymbol{\sigma}=\frac{\eta_{p}}{\lambda_{0}}(\boldsymbol{\tau}-\boldsymbol{I})$, and in turn, the conformation tensor $\boldsymbol{\tau}$ must be written as $\boldsymbol{\tau}=\exp (\boldsymbol{\psi})$ in the standard viscoelastic formulation detailed above, (2.1), (2.2) and (2.3). The new 
equations of the log-conformation formulation are now expressed as follows:

$$
\begin{array}{r}
\rho \frac{\partial \boldsymbol{u}}{\partial t}-\frac{\eta_{0}(1-\beta)}{\lambda_{0}} \nabla \cdot \exp (\boldsymbol{\psi})-2 \beta \eta_{0} \nabla \cdot\left(\nabla^{s} \boldsymbol{u}\right)+\rho \boldsymbol{u} \cdot \nabla \boldsymbol{u}+\nabla p=\boldsymbol{f} \\
\nabla \cdot \boldsymbol{u}=0, \\
\frac{1}{2 \lambda_{0}}(\exp (\boldsymbol{\psi})-\boldsymbol{I})-\nabla^{s} \boldsymbol{u} \\
+\frac{\lambda}{2 \lambda_{0}}\left(\frac{\partial \exp (\boldsymbol{\psi})}{\partial t}+\boldsymbol{u} \cdot \nabla \exp (\boldsymbol{\psi})-\exp (\boldsymbol{\psi}) \cdot \nabla \boldsymbol{u}-(\nabla \boldsymbol{u})^{T} \cdot \exp (\boldsymbol{\psi})+2 \nabla^{s} \boldsymbol{u}\right)=\mathbf{0}
\end{array}
$$

where the unknowns are the velocity, the pressure, and tensor $\boldsymbol{\psi}$, which depends directly on the viscoelastic stress tensor $\boldsymbol{\sigma}$.

This logarithm reformulation employed reminds of the formulation used by Coronado et al. in [15], although there the conformation tensor was simply replaced by $\exp (\boldsymbol{\psi})$. Another change was introduced in [15] with respect to the original log-conformation reformulation: the decomposition of the gradient of the velocity into three different tensors. However, this has not been taken into account in our formulation. In this sense, the modified log-conformation formulation proposed by Saramito [18] is very similar to our formulation.

Let us introduce some notation, useful in the next subsections. Calling now $\boldsymbol{U}=$ $[\boldsymbol{u}, p, \boldsymbol{\psi}]$ and $\boldsymbol{F}=\left[\boldsymbol{f}, 0, \frac{1}{2 \lambda_{0}} \boldsymbol{I}\right]$ and defining

$$
\begin{gathered}
\mathcal{D}_{t}(\boldsymbol{U}):=\left(\begin{array}{c}
\rho \frac{\partial \boldsymbol{u}}{\partial t} \\
0 \\
\frac{\lambda}{2 \lambda_{0}} \frac{\partial \exp (\boldsymbol{\psi})}{\partial t}
\end{array}\right), \\
\mathcal{L}(\hat{\boldsymbol{u}} ; \boldsymbol{U}):=\left(\begin{array}{c}
-\frac{\eta_{0}(1-\beta)}{\lambda_{0}} \nabla \cdot(\exp (\boldsymbol{\psi}))-2 \beta \eta_{0} \nabla \cdot\left(\nabla^{s} \boldsymbol{u}\right)+\rho \hat{\boldsymbol{u}} \cdot \nabla \boldsymbol{u}+\nabla p \\
\nabla \cdot \boldsymbol{u} \\
\frac{1}{2 \lambda_{0}} \exp (\boldsymbol{\psi})-\nabla^{s} \boldsymbol{u}+\frac{\lambda}{2 \lambda_{0}}(\hat{\boldsymbol{u}} \cdot \nabla(\exp (\boldsymbol{\psi})) \\
\left.-\exp (\boldsymbol{\psi}) \cdot \nabla \hat{\boldsymbol{u}}-(\nabla \hat{\boldsymbol{u}})^{T} \cdot \exp (\boldsymbol{\psi})+2 \nabla^{s} \boldsymbol{u}\right)
\end{array}\right),
\end{gathered}
$$

we may write (2.8)-(2.10) as

$$
\mathcal{D}_{t}(\boldsymbol{U})+\mathcal{L}(\boldsymbol{u} ; \boldsymbol{U})=\boldsymbol{F} .
$$

The notation $\hat{\boldsymbol{u}}$ in (2.12) is used to distinguish the different arguments in which the velocity appears. These equations need to be complemented with initial and boundary conditions to close the problem. For the sake of simplicity, in the exposition we only consider the simplest boundary condition $\boldsymbol{u}=\mathbf{0}$ on $\partial \Omega$ for all time. Boundary conditions for the $\boldsymbol{\psi}$ tensor will be similar to those for the elastic stresses $\boldsymbol{\sigma}$ in the standard formulation: they do not need to be prescribed, but imposing them can suppose a significant computational save. We will indicate in our examples where the boundary condition are prescribed.

The problem is completely defined by the initial conditions for the velocity and the new variable $\boldsymbol{\psi}$, which are denoted by $\boldsymbol{u}=\boldsymbol{u}^{0}$, and $\boldsymbol{\psi}=\boldsymbol{\psi}^{0}$ at time $t=0$, with $\boldsymbol{u}^{0}$ and $\boldsymbol{\psi}^{0}$ functions defined on the whole domain $\Omega$.

\subsection{Variational formulation}

In order to write the weak form of the problem, let us introduce some notation. The space of square integrable functions in a domain $\omega$ is denoted by $L^{2}(\omega)$, and the space of 
functions whose distributional derivatives of order up to $m \geq 0$ (integer) belong to $L^{2}(\omega)$ is denoted by $H^{m}(\omega)$.

The space $H_{0}^{1}(\omega)$ is made up of functions in $H^{1}(\omega)$ vanishing on $\partial \omega$. The topological dual of $H_{0}^{1}(\omega)$ is denoted by $H^{-1}(\Omega)$, the duality pairing being $\langle\cdot, \cdot\rangle$. The $L^{2}$ inner product in $\omega$ (for scalars, vectors and tensors) is denoted by $(\cdot, \cdot)_{\omega}$ and the integral over $\omega$ of the product of two general functions is written as $\langle\cdot, \cdot\rangle_{\omega}$, the subscript being omitted when $\omega=\Omega$. The norm in a space $X$ is denoted by $\|\cdot\|_{X}$, except in the case $X=L^{2}(\Omega)$, case in which the subscript is omitted.

Using this notation, velocity and pressure $\mathrm{FE}$ spaces for the continuous problem are $\boldsymbol{V}_{0}=H_{0}^{1}(\Omega)^{d}$ and $\mathcal{Q}=L^{2}(\Omega) / \mathbb{R}$, and the space for the tensor $\boldsymbol{\psi}$ is denoted by $\boldsymbol{\Upsilon}$ for each fixed time $t$, where an appropriate regularity is assumed. The weak form of the problem consists in finding $\boldsymbol{U}=[\boldsymbol{u}, p, \boldsymbol{\psi}]:\left(0, t_{\mathrm{f}}\right) \longrightarrow \mathcal{X}:=\boldsymbol{V}_{0} \times \boldsymbol{Q} \times \boldsymbol{\Upsilon}$, such that the initial conditions are satisfied and:

$$
\begin{aligned}
\left(\rho \frac{\partial \boldsymbol{u}}{\partial t}, \boldsymbol{v}\right)+\frac{\eta_{0}(1-\beta)}{\lambda_{0}}\left(\exp (\boldsymbol{\psi}), \nabla^{s} \boldsymbol{v}\right)+2\left(\beta \eta_{0} \nabla^{s} \boldsymbol{u}, \nabla^{s} \boldsymbol{v}\right) & \\
+\langle\rho \boldsymbol{u} \cdot \nabla \boldsymbol{u}, \boldsymbol{v}\rangle-(p, \nabla \cdot \boldsymbol{v}) & =\langle\boldsymbol{f}, \boldsymbol{v}\rangle, \\
(q, \nabla \cdot \boldsymbol{u}) & =0, \\
\frac{1}{2 \lambda_{0}}(\exp (\boldsymbol{\psi}), \boldsymbol{\chi})-\left(\nabla^{s} \boldsymbol{u}, \boldsymbol{\chi}\right)+\frac{\lambda}{2 \lambda_{0}}\left(\frac{\partial \exp (\boldsymbol{\psi})}{\partial t}, \boldsymbol{\chi}\right) & \\
+\frac{\lambda}{2 \lambda_{0}}(\boldsymbol{u} \cdot \nabla \exp (\boldsymbol{\psi}), \boldsymbol{\chi}) & \\
+\frac{\lambda}{2 \lambda_{0}}\left(-\exp (\boldsymbol{\psi}) \cdot \nabla \boldsymbol{u}-(\nabla \boldsymbol{u})^{T} \cdot \exp (\boldsymbol{\psi})+2 \nabla^{s} \boldsymbol{u}, \boldsymbol{\chi}\right) & =\frac{1}{2 \lambda_{0}}\langle\boldsymbol{I}, \boldsymbol{\chi}\rangle,
\end{aligned}
$$

for all $\boldsymbol{V}=[\boldsymbol{v}, q, \boldsymbol{\chi}] \in \mathcal{X}$, where it is assumed that $\boldsymbol{f}$ is such that $\langle\boldsymbol{f}, \boldsymbol{v}\rangle$ is well defined. In compact form, the problem can be written as:

$$
\left(\mathcal{D}_{t}(\boldsymbol{U}), \boldsymbol{V}\right)+B(\boldsymbol{u} ; \boldsymbol{U}, \boldsymbol{V})=L(\boldsymbol{V}),
$$

where

$$
\begin{aligned}
\left(\mathcal{D}_{t}(\boldsymbol{U}), \boldsymbol{V}\right) & =\left(\rho \frac{\partial \boldsymbol{u}}{\partial t}, \boldsymbol{v}\right)+\frac{\lambda}{2 \lambda_{0}}\left(\frac{\partial \exp (\boldsymbol{\psi})}{\partial t}, \boldsymbol{\chi}\right) \\
B(\hat{\boldsymbol{u}} ; \boldsymbol{U}, \boldsymbol{V}) & =\frac{\eta_{0}(1-\beta)}{\lambda_{0}}\left(\exp (\boldsymbol{\psi}), \nabla^{s} \boldsymbol{v}\right)+2\left(\beta \eta_{0} \nabla^{s} \boldsymbol{u}, \nabla^{s} \boldsymbol{v}\right)+\langle\rho \hat{\boldsymbol{u}} \cdot \nabla \boldsymbol{u}, \boldsymbol{v}\rangle-(p, \nabla \cdot \boldsymbol{v}) \\
& +(\nabla \cdot \boldsymbol{u}, q)+\frac{1}{2 \lambda_{0}}(\exp (\boldsymbol{\psi}), \boldsymbol{\chi})-\left(\nabla^{s} \boldsymbol{u}, \boldsymbol{\chi}\right) \\
& +\frac{\lambda}{2 \lambda_{0}}\left(\boldsymbol{u} \cdot \nabla \exp (\boldsymbol{\psi})-\exp (\boldsymbol{\psi}) \cdot \nabla \boldsymbol{u}-(\nabla \boldsymbol{u})^{T} \cdot \exp (\boldsymbol{\psi})+2 \nabla^{s} \boldsymbol{u}, \boldsymbol{\chi}\right) \\
L(\boldsymbol{V}) & =\langle\boldsymbol{f}, \boldsymbol{v}\rangle+\frac{1}{2 \lambda_{0}}\langle\boldsymbol{I}, \boldsymbol{\chi}\rangle .
\end{aligned}
$$

Note that the test function $\chi$ is, from the physical point of view, a stress, whereas $\boldsymbol{\psi}$ is the logarithm of the conformation tensor (and thus, dimensionless). We could also have used a test function for the constitutive equation of the form $\frac{\eta_{p}}{\lambda_{0}} \exp (\chi)$, where now $\chi$ would be dimensionless. This would simplify the analysis (some stability would follow taking $\chi=\boldsymbol{\psi}$ ), but complicate significantly the finite element approximations described below.

\subsection{Linearization of the exponential}

Apart from the typical non-linearities associated with the standard viscoelastic problem such as convective or stretching terms, now we have to consider how to process the exponential function of the tensor $\boldsymbol{\psi}$. It has been treated as follows:

$$
\exp (\boldsymbol{\psi})=\exp (\hat{\boldsymbol{\psi}}+\delta \boldsymbol{\psi})=\exp (\hat{\boldsymbol{\psi}}) \cdot \exp (\delta \boldsymbol{\psi}),
$$


where $\delta \boldsymbol{\psi}=\boldsymbol{\psi}-\hat{\boldsymbol{\psi}}$ is considered as the incremental part and $\hat{\boldsymbol{\psi}}$ is a known tensor, which will be calculated at the previous iteration in the linearization scheme. The term $\exp (\delta \boldsymbol{\psi})$ has been linearized in turn through a Taylor expansion with a truncation error of order $(\delta \boldsymbol{\psi})^{2}$. Therefore the approximation is defined as

$$
\exp (\delta \boldsymbol{\psi}) \approx \boldsymbol{I}+\delta \boldsymbol{\psi}
$$

Consequently,

$$
\exp (\boldsymbol{\psi}) \approx \exp (\hat{\boldsymbol{\psi}}) \cdot(\boldsymbol{I}+\delta \boldsymbol{\psi})=\exp (\hat{\boldsymbol{\psi}}) \cdot \boldsymbol{\psi}+\exp (\hat{\boldsymbol{\psi}}) \cdot(\boldsymbol{I}-\hat{\boldsymbol{\psi}}) .
$$

So, inserting the approximation into the system (2.8) - (2.10), the system is linearized around $\hat{\psi}$ as follows

$$
\begin{array}{r}
\rho \frac{\partial \boldsymbol{u}}{\partial t}-\frac{\eta_{0}(1-\beta)}{\lambda_{0}} \nabla \cdot(\exp (\hat{\boldsymbol{\psi}}) \cdot \boldsymbol{\psi})-2 \beta \eta_{0} \nabla \cdot\left(\nabla^{s} \boldsymbol{u}\right)+\rho \boldsymbol{u} \cdot \nabla \boldsymbol{u}+\nabla p \\
=\boldsymbol{f}+\frac{\eta_{0}(1-\beta)}{\lambda_{0}} \nabla \cdot(-\exp (\hat{\boldsymbol{\psi}}) \cdot \hat{\boldsymbol{\psi}}+\exp (\hat{\boldsymbol{\psi}})) \\
\nabla \cdot \boldsymbol{u}=0 \\
\frac{1}{2 \lambda_{0}} \exp (\hat{\boldsymbol{\psi}}) \cdot \boldsymbol{\psi}-\nabla^{s} \boldsymbol{u}+\frac{\lambda}{2 \lambda_{0}}\left(\frac{\partial(\exp (\hat{\boldsymbol{\psi}}) \cdot \boldsymbol{\psi})}{\partial t}+2 \nabla^{s} \boldsymbol{u}\right. \\
+\boldsymbol{u} \cdot \nabla(\exp (\hat{\boldsymbol{\psi}}) \cdot \boldsymbol{\psi}-\exp (\hat{\boldsymbol{\psi}}) \cdot \hat{\boldsymbol{\psi}}+\exp (\hat{\boldsymbol{\psi}})) \\
-(\exp (\hat{\boldsymbol{\psi}}) \cdot \boldsymbol{\psi}-\exp (\hat{\boldsymbol{\psi}}) \cdot \hat{\boldsymbol{\psi}}+\exp (\hat{\boldsymbol{\psi}})) \cdot \nabla \boldsymbol{u} \\
\left.-(\nabla \boldsymbol{u})^{T} \cdot(\exp (\hat{\boldsymbol{\psi}}) \cdot \boldsymbol{\psi}-\exp (\hat{\boldsymbol{\psi}}) \cdot \hat{\boldsymbol{\psi}}+\exp (\hat{\boldsymbol{\psi}}))\right) \\
=\frac{1}{2 \lambda_{0}}(\boldsymbol{I}-\exp (\hat{\boldsymbol{\psi}})+\exp (\hat{\boldsymbol{\psi}}) \cdot \hat{\boldsymbol{\psi}})+\frac{\lambda}{2 \lambda_{0}}\left(\frac{\partial(\exp (\hat{\boldsymbol{\psi}}) \cdot \hat{\boldsymbol{\psi}})}{\partial t}-\frac{\partial \exp (\hat{\boldsymbol{\psi}})}{\partial t}\right)
\end{array}
$$

The variational formulation of this problem is straightforward.

\subsection{Galerkin finite element discretization}

The standard Galerkin approximation for the variational problem, which has been established in (2.17), is described next. Let $\mathcal{T}_{h}=\{K\}$ be a FE partition of the domain $\Omega$. The diameter of an element $K \in \mathcal{T}_{h}$ is denoted by $h_{K}$ and the diameter of the partition is defined as $h=\max \left\{h_{K} \mid K \in \mathcal{T}_{h}\right\}$. From $\mathcal{T}_{h}$ we may construct conforming FE spaces for the velocity, the pressure and the elastic stress, $\mathcal{V}_{h} \subset \mathcal{V}, \mathcal{Q}_{h} \subset \mathcal{Q}, \boldsymbol{\Upsilon}_{h} \subset \boldsymbol{\Upsilon}$, respectively. Calling $\mathcal{X}_{h}:=\mathcal{V}_{h} \times \mathcal{Q}_{h} \times \Upsilon_{h}$, the Galerkin FE approximation of the problem consists in finding $\boldsymbol{U}_{h}:\left(0, t_{\mathrm{f}}\right) \longrightarrow \boldsymbol{\mathcal { X }}_{h}$, such that:

$$
\left(\mathcal{D}_{t}\left(\boldsymbol{U}_{h}\right), \boldsymbol{V}_{h}\right)+B\left(\boldsymbol{u}_{h} ; \boldsymbol{U}_{h}, \boldsymbol{V}_{h}\right)=L\left(\boldsymbol{V}_{h}\right),
$$

for all $\boldsymbol{V}_{h}=\left[\boldsymbol{v}_{h}, q_{h}, \boldsymbol{\chi}_{h}\right] \in \mathcal{X}_{h}$, and satisfying the appropriate initial conditions.

\subsection{Monolithic time discretization}

For the time discretization, we have used a monolithic approach, although it would also be possible to employ a fractional step technique, as in [35]. There are a lot of possibilities for the discretization in time, but we will restrict ourselves to the classical backward difference (BDF) approximations.

Consider a partition of the interval $\left[0, t_{\mathrm{f}}\right]$ into $m$ subintervals of constant size $\delta t$, and let $f(t)$ be a generic time-dependent function. We will denote as $f^{n}$ the approximation to 
$f\left(t^{n}\right)$, with $t^{n}=n \delta t, n=0,1,2, \ldots, m$. A BDF approximation to the time derivative of the function $f$ order $k=1,2, \ldots$, is given by $\frac{\delta_{k} f^{n+1}}{\delta t}$, where $\delta_{k} f^{n+1}$ is defined as

$$
\delta_{k} f^{n+1}=\frac{1}{\gamma_{k}}\left(f^{n+1}-\sum_{i=0}^{k-1} \varphi_{k}^{i} f^{n-i}\right),
$$

and where $\gamma_{k}$ and $\varphi_{k}^{i}$ are parameters. In particular, since the time evolution is not the main emphasis of this work, in the numerical examples we have used the simplest BDF1 scheme (in fact, as a means to reach the stationary solution):

$$
\frac{\delta_{1} f^{n+1}}{\delta t}=\frac{f^{n+1}-f^{n}}{\delta t}=\left.\frac{\partial f}{\partial t}\right|_{t^{n+1}}+\mathcal{O}(\delta t) .
$$

A remark is needed for the time derivative of the exponential. Using approximation $(2.21)$, it is easily shown that the operations "linearization" and "time approximation" commute if we identify $\hat{\boldsymbol{\psi}}^{n}=\boldsymbol{\psi}^{n}$. Indeed, in both cases we obtain:

$$
\begin{aligned}
\left.\frac{\partial(\exp (\boldsymbol{\psi}))}{\partial t}\right|_{t^{n+1}} & =\frac{1}{\delta t}\left[\exp \left(\hat{\boldsymbol{\psi}}^{n+1}\right) \cdot \boldsymbol{\psi}^{n+1}+\exp \left(\hat{\boldsymbol{\psi}}^{n+1}\right)-\exp \left(\hat{\boldsymbol{\psi}}^{n+1}\right) \cdot \hat{\boldsymbol{\psi}}^{n+1}\right. \\
& \left.-\exp \left(\boldsymbol{\psi}^{n}\right)\right]+\mathcal{O}(\delta t)+\mathcal{O}\left(\left(\delta \boldsymbol{\psi}^{n+1}\right)^{2}\right)
\end{aligned}
$$

\section{Design of a stable finite element formulation}

\subsection{Residual based stabilized finite element method}

We present in this section two stabilized finite element formulations for computing viscoelastic flows using the logarithm constitutive reformulation, in particular applied to the Oldroyd-B constitutive model.

VMS methods consist in the splitting of the unknown $\boldsymbol{U}$ in a component $\boldsymbol{U}_{h}$, which can be resolved by the FE space, and the remainder $\tilde{\boldsymbol{U}}$, that will be called sub-grid scale. The framework is based on the work by Hughes et al. [26]. In the context of a three field formulation for flow problems, see [36] and [2]. The sub-grid scale needs to be approximated in a simple manner, with the goal of capturing its effect and yielding a stable formulation. The final number of degrees of freedom is the same as the Galerkin method. Different approaches can be followed to approximate the sub-scale and to choose the space where it is defined.

The problem we wish to approximate is (2.13) in differential form and (2.17) in variational form. Suppose for the moment that $\mathcal{L}(\hat{\boldsymbol{u}} ; \cdot)$ is a linear operator (for $\hat{\boldsymbol{u}}$ given). After introducing the subscales decomposition and integrating by parts, the VMS method leads to the problem of finding $\boldsymbol{U}_{h}:\left(0, t_{\mathrm{f}}\right) \longrightarrow \boldsymbol{X}_{h}$ such that

$$
\left(\mathcal{D}_{t}\left(\boldsymbol{U}_{h}\right), \boldsymbol{V}_{h}\right)+B\left(\boldsymbol{u}_{h} ; \boldsymbol{U}_{h}, \boldsymbol{V}_{h}\right)+\sum_{K}\left\langle\tilde{\boldsymbol{U}}, \mathcal{L}^{*}\left(\boldsymbol{u}_{h} ; \boldsymbol{V}_{h}\right)\right\rangle_{K}=L\left(\boldsymbol{V}_{h}\right),
$$

for all $\boldsymbol{V}_{h} \in \mathcal{X}_{h}$, where $\mathcal{L}^{*}(\hat{\boldsymbol{u}} ; \cdot)$ is the formal adjoint operator of $\mathcal{L}(\hat{\boldsymbol{u}} ; \cdot)$ and $\tilde{\boldsymbol{U}}$ is the subgrid scale, which needs to be approximated, without considering boundary conditions.

Let us remark that two different schemes can be followed at this point to get the stabilized problem:

1. Since operator $\mathcal{L}(\hat{\boldsymbol{u}} ; \cdot)$ in $(2.13)$ is in fact non-linear because of the exponential of $\boldsymbol{\psi}$, the VMS strategy needs to be applied to a certain linearization. The one described previously could be used, but other options are also possible. 
2. The alternative is to work with the standard form of the problem (2.5) (depending on the elastic stress $\boldsymbol{\sigma})$, in which operator $\mathcal{L}_{0}(\hat{\boldsymbol{u}} ; \cdot)$ is linear, design the stabilized FE problem based on the VMS concept (involving operator $\mathcal{L}_{0}^{*}(\hat{\boldsymbol{u}} ; \cdot)$ ) and then change variables to obtain a log-conformation formulation.

Both cases give us different stabilized formulations, although for simplicity only the second one has been considered in this work. Therefore, the adjoint operator we need to consider is

$$
\mathcal{L}_{0}^{*}(\hat{\boldsymbol{u}} ; \boldsymbol{U}):=\left(\begin{array}{c}
\nabla \cdot \boldsymbol{\chi}-2 \beta \eta_{0} \nabla \cdot\left(\nabla^{s} \boldsymbol{v}\right)-\rho \hat{\boldsymbol{u}} \cdot \nabla \boldsymbol{v}-\nabla q \\
-\nabla \cdot \boldsymbol{v} \\
\frac{1}{2 \eta_{0}(1-\beta)} \boldsymbol{\chi}+\nabla^{s} \boldsymbol{v}-\frac{\lambda}{2 \eta_{0}(1-\beta)}\left(\hat{\boldsymbol{u}} \cdot \nabla \boldsymbol{\chi}+\boldsymbol{\chi} \cdot(\nabla \hat{\boldsymbol{u}})^{T}+\nabla \hat{\boldsymbol{u}} \cdot \boldsymbol{\chi}\right)
\end{array}\right) .
$$

Taking $\tilde{P}$ as the $L^{2}$ projection onto the space of sub-grid scales, the approximation we consider for the sub-grid scales within each element is

$$
\tilde{\boldsymbol{U}}=\boldsymbol{\alpha} \tilde{P}\left[\boldsymbol{F}-\mathcal{D}_{t}\left(\boldsymbol{U}_{h}\right)-\mathcal{L}\left(\boldsymbol{u}_{h} ; \boldsymbol{U}_{h}\right)\right],
$$

where $\boldsymbol{\alpha}$ is a diagonal matrix $\boldsymbol{\alpha}=\operatorname{diag}\left(\alpha_{1} \boldsymbol{I}_{d}, \alpha_{2}, \alpha_{3} \boldsymbol{I}_{d \times d}\right)$ with $\boldsymbol{I}_{d}$ the identity on vectors of $\mathbb{R}^{d}, \boldsymbol{I}_{d \times d}$ the identity on second order tensor and the parameters $\alpha_{i}, i=1,2,3$ are computed as

$$
\begin{aligned}
& \alpha_{1}=\left[c_{1} \frac{\eta_{0}}{h_{1}^{2}}+c_{2} \frac{\rho\left|\boldsymbol{u}_{h}\right|}{h_{2}}\right]^{-1} \\
& \alpha_{2}=\frac{h_{1}^{2}}{c_{1} \alpha_{1}} \\
& \alpha_{3}=\left[c_{3} \frac{1}{2 \eta_{0}(1-\beta)}+c_{4}\left(\frac{\lambda}{2 \eta_{0}(1-\beta)} \frac{\left|\boldsymbol{u}_{h}\right|}{h_{2}}+\frac{\lambda}{\eta_{0}(1-\beta)}\left|\nabla \boldsymbol{u}_{h}\right|\right)\right]^{-1},
\end{aligned}
$$

where $h_{1}$ corresponds to a characteristic element length calculated in the two-dimensional case as the square root of the element area, and in three-dimensional case as the cubic root of the element volume, whereas $h_{2}$ represents the characteristic length associated to the element length in the streamline direction. On the other side, $\left|\boldsymbol{u}_{h}\right|$ is the Euclidean norm of the velocity while $\left|\nabla \boldsymbol{u}_{h}\right|$ is calculated by means of the Frobenious norm. The dimensionless constants $c_{i}, i=1, . ., 4$ are algorithmic parameters in the formulation, and the values adopted in this work are $c_{1}=4.0, c_{2}=2.0, c_{3}=4.0, c_{4}=0.25$ for linear elements. Numerical analysis indicates that they have to be of order one [36], and that $c_{4}<1$. Moreover, $c_{1}=4.0, c_{2}=2.0$ are the optimal values for the approximation of the one-dimensional convection-diffusion equation. Note that the values for these constants are the same as those used for the standard formulation [2].

Inserting (3.3) in (3.1), with $\boldsymbol{\alpha}$ given above and using the adjoint operator (3.2), we obtain the following problem: find $\boldsymbol{U}_{h} \in \mathcal{X}_{h}$ such that

$$
\begin{aligned}
\left(\mathcal{D}_{t}\left(\boldsymbol{U}_{h}\right), \boldsymbol{V}_{h}\right)+B\left(\boldsymbol{u}_{h} ; \boldsymbol{U}_{h}, \boldsymbol{V}_{h}\right) & +S_{1}\left(\boldsymbol{u}_{h} ; \boldsymbol{U}_{h}, \boldsymbol{V}_{h}\right)+S_{2}\left(\boldsymbol{U}_{h}, \boldsymbol{V}_{h}\right)+S_{3}\left(\boldsymbol{u}_{h} ; \boldsymbol{U}_{h}, \boldsymbol{V}_{h}\right) \\
& =L\left(\boldsymbol{V}_{h}\right)+R_{1}\left(\boldsymbol{u}_{h} ; \boldsymbol{V}_{h}\right)+R_{3}\left(\boldsymbol{u}_{h} ; \boldsymbol{V}_{h}\right),
\end{aligned}
$$


for all $\boldsymbol{V}_{h} \in \mathcal{X}_{h}$, where

$$
\begin{aligned}
S_{1}\left(\hat{\boldsymbol{u}}_{h} ; \boldsymbol{U}_{h}, \boldsymbol{V}_{h}\right)= & \sum_{K} \alpha_{1}\left\langle\tilde { P } \left[\rho \frac{\partial \boldsymbol{u}_{h}}{\partial t}-\frac{\eta_{0}(1-\beta)}{\lambda_{0}} \nabla \cdot\left(\exp \left(\boldsymbol{\psi}_{h}\right)\right)-2 \beta \eta_{0} \nabla \cdot\left(\nabla^{s} \boldsymbol{u}_{h}\right)\right.\right. \\
& \left.\left.+\rho \hat{\boldsymbol{u}}_{h} \cdot \nabla \boldsymbol{u}_{h}+\nabla p_{h}\right],-\nabla \cdot \boldsymbol{\chi}_{h}+2 \beta \eta_{0} \nabla \cdot\left(\nabla^{s} \boldsymbol{v}_{h}\right)+\rho \hat{\boldsymbol{u}}_{h} \cdot \nabla \boldsymbol{v}_{h}+\nabla q_{h}\right\rangle_{K}, \\
S_{2}\left(\boldsymbol{U}_{h}, \boldsymbol{V}_{h}\right)= & \sum_{K} \alpha_{2}\left\langle\tilde{P}\left[\nabla \cdot \boldsymbol{u}_{h}\right], \nabla \cdot \boldsymbol{v}_{h}\right\rangle_{K}, \\
S_{3}\left(\hat{\boldsymbol{u}}_{h} ; \boldsymbol{U}_{h}, \boldsymbol{V}_{h}\right)= & \sum_{K} \alpha_{3}\left\langle\tilde { P } \left[\frac{1}{2 \lambda_{0}} \exp \left(\boldsymbol{\psi}_{h}\right)-\nabla^{s} \boldsymbol{u}_{h}+\frac{\lambda}{2 \lambda_{0}}\left(\frac{\partial\left(\exp \left(\boldsymbol{\psi}_{h}\right)\right)}{\partial t}+\hat{\boldsymbol{u}}_{h} \cdot \nabla\left(\exp \left(\boldsymbol{\psi}_{h}\right)\right)\right)\right.\right. \\
& \left.-\exp \left(\boldsymbol{\psi}_{h}\right) \cdot \nabla \hat{\boldsymbol{u}}_{h}-\left(\nabla \hat{\boldsymbol{u}}_{h}\right)^{T} \cdot \exp \left(\boldsymbol{\psi}_{h}\right)+2 \nabla^{s} \boldsymbol{u}_{h}\right], \\
& -\frac{1}{2 \eta_{0}(1-\beta)} \boldsymbol{\chi}_{h}-\nabla^{s} \boldsymbol{v}_{h} \\
& \left.+\frac{\lambda}{2 \eta_{0}(1-\beta)}\left(\hat{\boldsymbol{u}}_{h} \cdot \nabla \boldsymbol{\chi}_{h}+\boldsymbol{\chi}_{h} \cdot\left(\nabla \hat{\boldsymbol{u}}_{h}\right)^{T}+\nabla \hat{\boldsymbol{u}}_{h} \cdot \boldsymbol{\chi}_{h}\right)\right\rangle_{K}, \\
R_{1}\left(\hat{\boldsymbol{u}}_{h} ; \boldsymbol{V}_{h}\right)= & \sum_{K} \alpha_{1}\left\langle\tilde{P}[\boldsymbol{f}],-\nabla \cdot \boldsymbol{\chi}_{h}+2 \beta \eta_{0} \nabla \cdot\left(\nabla^{s} \boldsymbol{v}_{h}\right)+\rho \hat{\boldsymbol{u}}_{h} \cdot \nabla \boldsymbol{v}_{h}+\nabla q_{h}\right\rangle_{K}, \\
R_{3}\left(\hat{\boldsymbol{u}}_{h} ; \boldsymbol{V}_{h}\right)= & \sum_{K} \alpha_{3}\left\langle\tilde{P}\left[\frac{1}{2 \lambda_{0}} \boldsymbol{I}\right],-\frac{1}{2 \eta_{0}(1-\beta)} \boldsymbol{\chi}_{h}-\nabla^{s} \boldsymbol{v}_{h},\right. \\
& \left.+\frac{\lambda}{2 \eta_{0}(1-\beta)}\left(\hat{\boldsymbol{u}}_{h} \cdot \nabla \boldsymbol{\chi}_{h}+\boldsymbol{\chi}_{h} \cdot\left(\nabla \hat{\boldsymbol{u}}_{h}\right)^{T}+\nabla \hat{\boldsymbol{u}}_{h} \cdot \boldsymbol{\chi}_{h}\right)\right\rangle_{K} \cdot
\end{aligned}
$$

In these equations, $\tilde{P}$ is the projection restricted to the appropriate components of the FE residual $\boldsymbol{R}_{h}:=\boldsymbol{F}-\mathcal{L}\left(\boldsymbol{u}_{h} ; \boldsymbol{U}_{h}\right)$ onto the space of sub-grid scales. It remains only to define the projection, for which we consider two possibilities. If we consider $\tilde{P}=I$ (identity), then the method is called Algebraic Sub-Grid Scales (ASGS). In the case that $\tilde{P}=P_{h}^{\perp}=I-P_{h}$ (where $P_{h}$ is the $L^{2}$ projection onto the appropriate finite element space), the name of the method is Orthogonal Sub-Grid Scales (OSS). Independently of the choice of the projection $\tilde{P}$, method (3.4) is consistent, since the terms added to the Galerkin ones are proportional to the FE residual $\boldsymbol{R}_{h}$.

\subsection{Split-OSS}

Method (3.4) is stable for smooth solutions, and displays the appropriate order of convergence, both for $\tilde{P}=I$ and for $\tilde{P}=P_{h}^{\perp}$. As it is indicated in [2], the OSGS method seems in general more accurate, whereas ASGS is cheaper because projections are not needed and it is more robust.

If we consider the case $\tilde{P}=P_{h}^{\perp}$, from (3.4) we can design a simplified method, which consists in neglecting the cross local inner-product terms, as well as some other terms that do not contribute to stability.

Following the considerations made in [37] for the construction of the Split OSGS stabilization for the traditional viscoelastic formulation, the modified method we propose for the log-conformation reformulation is: find $\boldsymbol{U}_{h}:\left(0, t_{\mathrm{f}}\right) \longrightarrow \mathcal{X}_{h}$ satisfying the appropriate initial conditions and such that

$$
\begin{aligned}
\left(\mathcal{D}_{t}\left(\boldsymbol{U}_{h}\right), \boldsymbol{V}_{h}\right)+B\left(\boldsymbol{u}_{h}, \boldsymbol{\psi}_{h} ; \boldsymbol{U}_{h}, \boldsymbol{V}_{h}\right)+S_{1}^{\perp}\left(\boldsymbol{u}_{h} ; \boldsymbol{U}_{h}, \boldsymbol{V}_{h}\right)+S_{2}^{\perp}\left(\boldsymbol{U}_{h}, \boldsymbol{V}_{h}\right) & \\
& +S_{3}^{\perp}\left(\boldsymbol{u}_{h} ; \boldsymbol{U}_{h}, \boldsymbol{V}_{h}\right)=L\left(\boldsymbol{V}_{h}\right),
\end{aligned}
$$


for all $\boldsymbol{V}_{h} \in \mathcal{X}_{h}$, where

$$
\begin{aligned}
& S_{1}^{\perp}\left(\hat{\boldsymbol{u}}_{h} ; \boldsymbol{U}_{h}, \boldsymbol{V}_{h}\right)=\sum_{K} \alpha_{1}\left\langle P_{h}^{\perp}\left[-\frac{\eta_{0}(1-\beta)}{\lambda_{0}} \nabla \cdot(\exp (\boldsymbol{\psi}))\right],-\nabla \cdot \boldsymbol{\chi}_{h}\right\rangle_{K} \\
& \quad+\sum_{K} \alpha_{1}\left\langle P_{h}^{\perp}\left[\nabla p_{h}\right], \nabla q_{h}\right\rangle_{K}+\sum_{K} \alpha_{1}\left\langle P\left[\rho \hat{\boldsymbol{u}}_{h} \cdot \nabla \boldsymbol{u}_{h}\right], \rho \hat{\boldsymbol{u}}_{h} \cdot \nabla \boldsymbol{v}_{h}\right\rangle_{K} \\
& S_{2}^{\perp}\left(\boldsymbol{U}_{h}, \boldsymbol{V}_{h}\right)=\sum_{K} \alpha_{2}\left\langle P_{h}^{\perp}\left[\nabla \cdot \boldsymbol{u}_{h}\right], \nabla \cdot \boldsymbol{v}_{h}\right\rangle_{K}, \\
& S_{3}^{\perp}\left(\hat{\boldsymbol{u}}_{h} ; \boldsymbol{U}_{h}, \boldsymbol{V}_{h}\right)=\sum_{K} \alpha_{3}\left\langle P_{h}^{\perp}\left[\nabla^{s} \boldsymbol{u}_{h}\right], \nabla^{s} \boldsymbol{v}_{h}\right\rangle_{K} \\
& \quad+\frac{\lambda^{2}}{4 \lambda_{0} \eta_{0}(1-\beta)} \sum^{2} \alpha_{3}\left\langleP _ { h } ^ { \perp } \left[\left(\hat{\boldsymbol{u}}_{h} \cdot \nabla\left(\exp \left(\boldsymbol{\psi}_{h}\right)\right], \hat{\boldsymbol{u}}_{h} \cdot \nabla \boldsymbol{\chi}_{h}\right\rangle_{K}\right.\right. \\
& \quad+\frac{\lambda^{2}}{4 \lambda_{0} \eta_{0}(1-\beta)} \sum_{K} \alpha_{3}\left\langleP _ { h } ^ { \perp } \left[-\exp \left(\boldsymbol{\psi}_{h}\right) \cdot \nabla \hat{\boldsymbol{u}}_{h}\right.\right. \\
& \left.\left.\left.\quad-\left(\nabla \hat{\boldsymbol{u}}_{h}\right)^{T} \cdot \exp \left(\boldsymbol{\psi}_{h}\right)+2 \nabla^{s} \boldsymbol{u}_{h}\right)\right],\left(\boldsymbol{\chi}_{h} \cdot\left(\nabla \hat{\boldsymbol{u}}_{h}\right)^{T}+\nabla \hat{\boldsymbol{u}}_{h} \cdot \boldsymbol{\chi}_{h}\right)\right\rangle_{K} \cdot
\end{aligned}
$$

Method (3.10) is not just a simplification of (3.4). For smooth solutions, both have an optimal convergence rate in $h$. However, in problems where the solution has strong gradients, we have found (3.10) more robust, similarly to what it is explained in [37].

As insignificant differences between the use of $S_{3}\left(\hat{\boldsymbol{u}}_{h} ; \boldsymbol{U}_{h}, \boldsymbol{V}_{h}\right)$ in (3.7) or the last modification indicated $S_{3}^{\perp}\left(\hat{\boldsymbol{u}}_{h} ; \boldsymbol{U}_{h}, \boldsymbol{V}_{h}\right)$ in (3.13) have been found, in the implementation we have taken (3.7) instead of (3.13). Therefore, we will refer to S-ASGS when $\tilde{P}=I$ in (3.7), and S-OSGS for the case $\tilde{P}=P_{h}^{\perp}=I-P_{h}$. However note that in both cases (3.11) and (3.12) have been used instead of (3.5) and (3.6), respectively. Note that the last term in (3.13) is unnecessary for stability and could be omitted.

\subsection{Linearized problem and algorithm}

The equations for incompressible viscoelastic flows have a high number of non-linear terms, in particular in the momentum and the constitutive equation. Obviously, these are maintained with the logarithmic reformulation, and furthermore, new non-linearities arising from the exponential function appear, as it has been exposed in Section 2.2.

For the convective term of the momentum equation we can use a fixed point scheme or Newton-Raphson's scheme. However, for the non-linear terms in the constitutive equation, we have used a Newton-Raphson linearization always, and it has been decisive to be able to compute some high Weissenberg cases and get the optimal convergence of the method. At each iteration of each time step, the equations written in Algorithm 1 are computed.

Let us make the following remarks about the algorithm used:

- The nonlinear term in the momentum equation can be linearized with the fixed point scheme or with Newton-Raphson's method, but in the algorithm presented the method used is the second.

- The exponential terms that appear both in the momentum equation and in the constitutive equation have been linearized using (2.21), taking the tensor $\hat{\boldsymbol{\psi}}$ as the one obtained from the previous iteration of the current time step.

- The computation of the exponential function, the gradient and the divergence of the exponential function of the variable $\boldsymbol{\psi}_{h}$ at the previous iteration must be calculated at each iteration. 
- All non-linear terms belonging to the constitutive equation, both in the Galerkin terms and in the stabilization, have been linearized using Newton-Raphson's method.

- Stabilization parameters are computed with the values of the unknowns at the previous iterations.

- The iterative treatment of the orthogonal projection is coupled to the linearization of the total system. Specifically, the orthogonal projection of any function $f$ has been approximated as $P_{h}^{\perp}\left(f^{i}\right) \approx f^{i}-P_{h}\left(f^{i-1}\right)$, the superscript being the iteration counter.

- Note that the ASGS method associated to the constitutive equation is considered when $\tilde{P}=I$, while the OSS is applied when $\tilde{P}=P_{h}^{\perp}$.

Apart from the linearization carried out in some terms just explained, we have found extremely useful the application of other techniques that lead to a better convergence. One of them is the under-relaxation scheme, taking as a relaxation parameter $\epsilon=0.5$, which has been found effective in most of the cases; the second tool employed is the continuation method in terms of the relaxation time $\lambda$, which consists in $N_{\lambda}$ continuation steps of equal size $\delta \lambda=\lambda / N_{\lambda}$. Note that continuation techniques can be employed because of the modification of the log-conformation formulation; if the original logarithmic formulation had been taken, they could not be used. Besides, the continuation loop and the linearization loop are coupled in the algorithm used.

In the equations displayed in the Algorithm 1 variables $\boldsymbol{u}_{h}^{j+1, i}, p_{h}^{j+1, i}, \boldsymbol{\psi}_{h}^{j+1, i}$ corresponding to the $(j+1)$ time step are denoted by $\boldsymbol{u}_{h}^{i}, p_{h}^{i}, \boldsymbol{\psi}_{h}^{i}$ for simplicity. Regarding the temporal terms, the notation introduced in Section 2.6 is employed here.

The equations considered in Algorithm 1 are solved inside of a general algorithm, presented in Algorithm 2, where all considerations made are taken into account.

\section{Numerical results}

In this section we will show some numerical examples where the benefits of the stabilized formulations applied to the logarithmic formulation will be demonstrated. As it is remarked in Section 2.6, most of the results shown have been obtained solving the steady problem directly, although many references and previous approaches with the logconformation were time-dependent.

The first result presented in Section 4.1 is a convergence test that will show the accuracy of the formulation. It is useful to show that it is optimally convergent for smooth solutions. Secondly, in Section 4.2, the well-known benchmark flow past a cylinder is tested to compare different quantities, such as the stress tensor around and downstream of the cylinder or the drag coefficient, with the values published in the literature. In Section 4.3 we present the classical 4:1 planar contraction flow problem for two different Reynolds numbers, $R e=1.0$ and $R e=0.0$, with the purpose of validating the results with a number of references. Finally, the last example in Section 4.4 is a three dimensional problem, designed as an extension of the two dimensional 4:1 contraction benchmark.

\subsection{Convergence test}

This first numerical results belong to the convergence study of the stabilized formulations employed for the log-conformation problem. The exact solution will be defined by the 


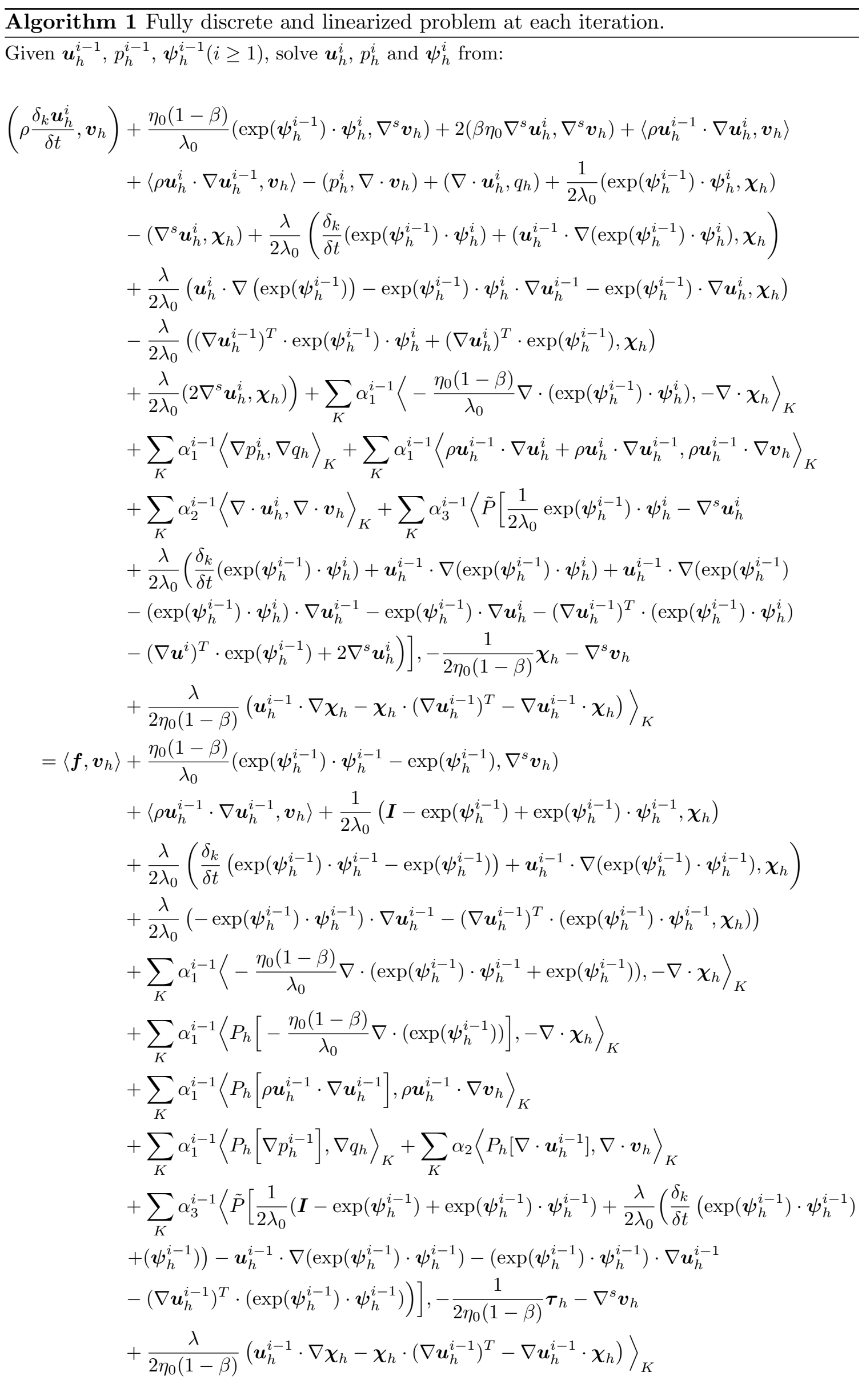




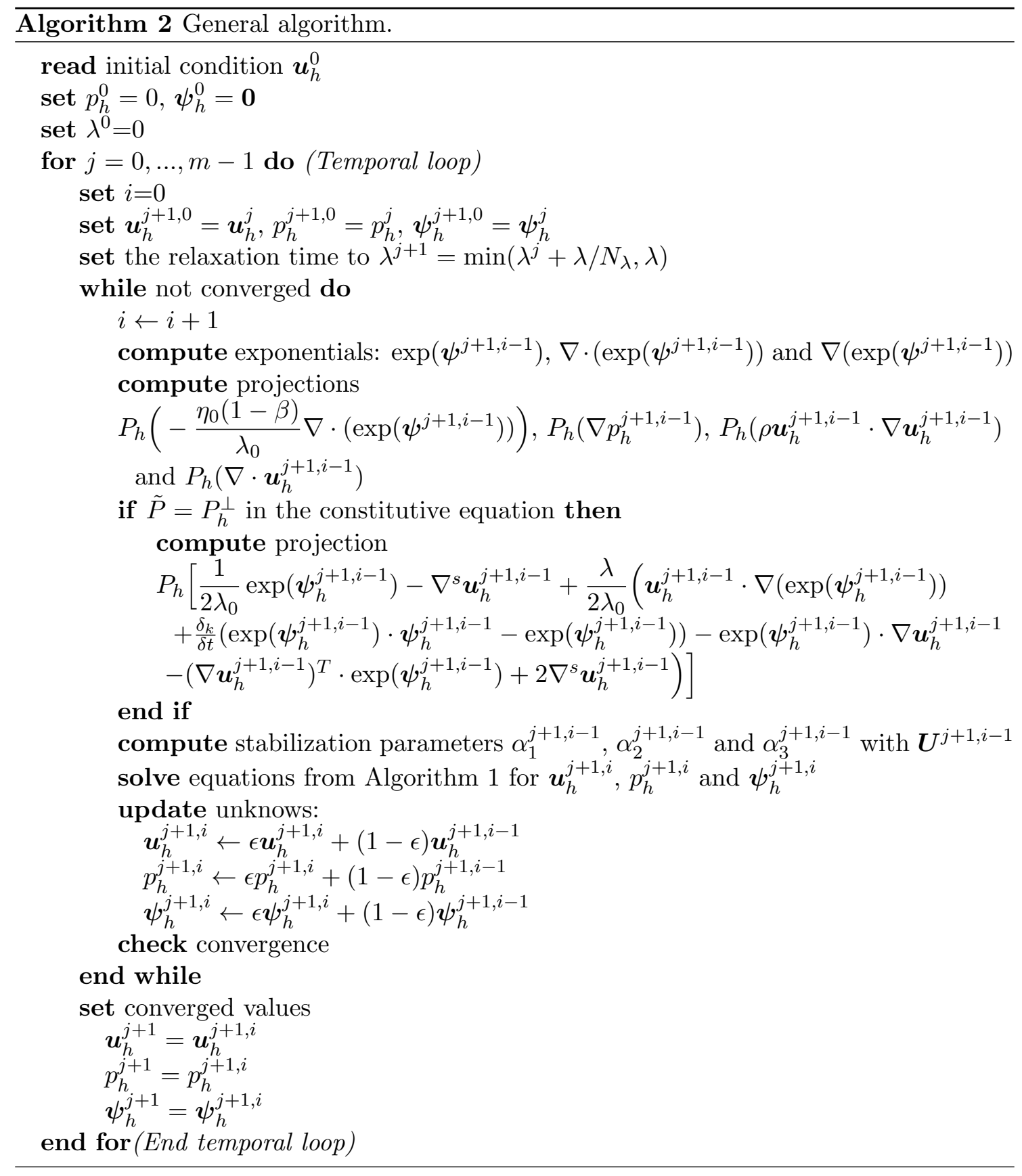


next set of functions:

$$
\begin{aligned}
u_{x}(x, y) & =2 x^{2} y(x-1)^{2}(y-1)(2 y-1) \\
u_{y}(x, y) & =-2 x y^{2}(x-1)(y-1)^{2}(2 x-1), \\
p(x, y) & =\sin (2 \pi x) \sin (2 \pi y) \\
\sigma_{x x}(x, y) & =5 \sin (2 \pi x) \sin (2 \pi y) \\
\sigma_{y y}(x, y) & =-5 \sin (2 \pi x) \sin (2 \pi y) \\
\sigma_{x y}(x, y) & =\sin (2 \pi x) \sin (2 \pi y)
\end{aligned}
$$

where the $x$ and $y$ components of the velocity and the stress tensor have been indicated with a subscript.

In order to satisfy the constitutive equation (2.10) with the velocity and the tensor $\boldsymbol{\psi}$, we have to add the forcing term

$$
\begin{aligned}
\boldsymbol{f}_{c}= & \frac{1}{2 \lambda_{0}}(\exp (\boldsymbol{\psi})-\boldsymbol{I})-\nabla^{s} \boldsymbol{u} \\
& +\frac{\lambda}{2 \lambda_{0}}\left(\boldsymbol{u} \cdot \nabla \exp (\boldsymbol{\psi})-\exp (\boldsymbol{\psi}) \cdot \nabla \boldsymbol{u}-(\nabla \boldsymbol{u})^{T} \cdot \exp (\boldsymbol{\psi})+2 \nabla^{s} \boldsymbol{u}\right)
\end{aligned}
$$

to the right-hand-side of the constitutive equation, with $\boldsymbol{u}$ and $\boldsymbol{\psi}$ being the given manufactured solution. Note that tensor $\boldsymbol{\psi}$ is obtained analytically from the stress tensor through the relation defined in Section 2.2.

The computational domain is the unit square, discretized using uniform structured meshes of bilinear $\left(Q_{1}\right)$ and biquadratic $\left(Q_{2}\right)$ quadrilateral elements. The range of element sizes employed in this study ranges between $h=0.003125$ and $h=0.0125$ for $Q_{1}$ elements, and between $h=0.00625$ and $h=0.025$ for $Q_{2}$ elements.

We have considered three different Weissenberg numbers for every mesh, We $=0.0$, $\mathrm{We}=0.5$ and $\mathrm{We}=1.0$. These quantities have been calculated with the maximum velocity value as characteristic velocity and the side of the square as characteristic length, taking into account that the expression of the dimensionless number is $\mathrm{We}=\frac{\lambda U}{L}$.

Regarding the optimal convergence rate expected, when the mesh is composed of linear elements, it is 2 in velocity and 1 in pressure and $\boldsymbol{\psi}$ for the $L^{2}$-norm, but using quadratic elements it is 3 in velocity and 2 in pressure and $\boldsymbol{\psi}$.

For both formulations, S-OSGS and S-ASGS, results are extremely close, so we have only represented represented the S-ASGS results in Fig. 1

\subsection{Viscoelastic fluid flow past a cylinder in a channel}

In the literature, we can find that this problem has been worked out using both formulations, the log-conformation one and the standard one. The most relevant results obtained with the standard formulation belong to Fan et al. [38] and Alves et al. [39]. In both works results are highly accurate, although their schemes failed for a Weissenberg number around 1. Other authors, who have published results with diverse techniques for the standard formulation, have obtained similar results regarding the numerical breakdown at relatively moderate Weissenberg numbers with an Oldroyd-B fluid, such as Caola et al. [40], Owens et al. [41], or Castillo et al. [2]. Nevertheless, the logarithmic reformulation of the equations allows us to get solutions for higher Weissenberg numbers. We will compare our results with the solutions found in the literature.

\subsubsection{Set up}

The geometry of a viscoelastic fluid flow past a confined cylinder in a channel considered in this section is depicted in Fig. 2. Note that only the half domain has been taken, for symmetry reasons. 
$Q_{1}$ elements
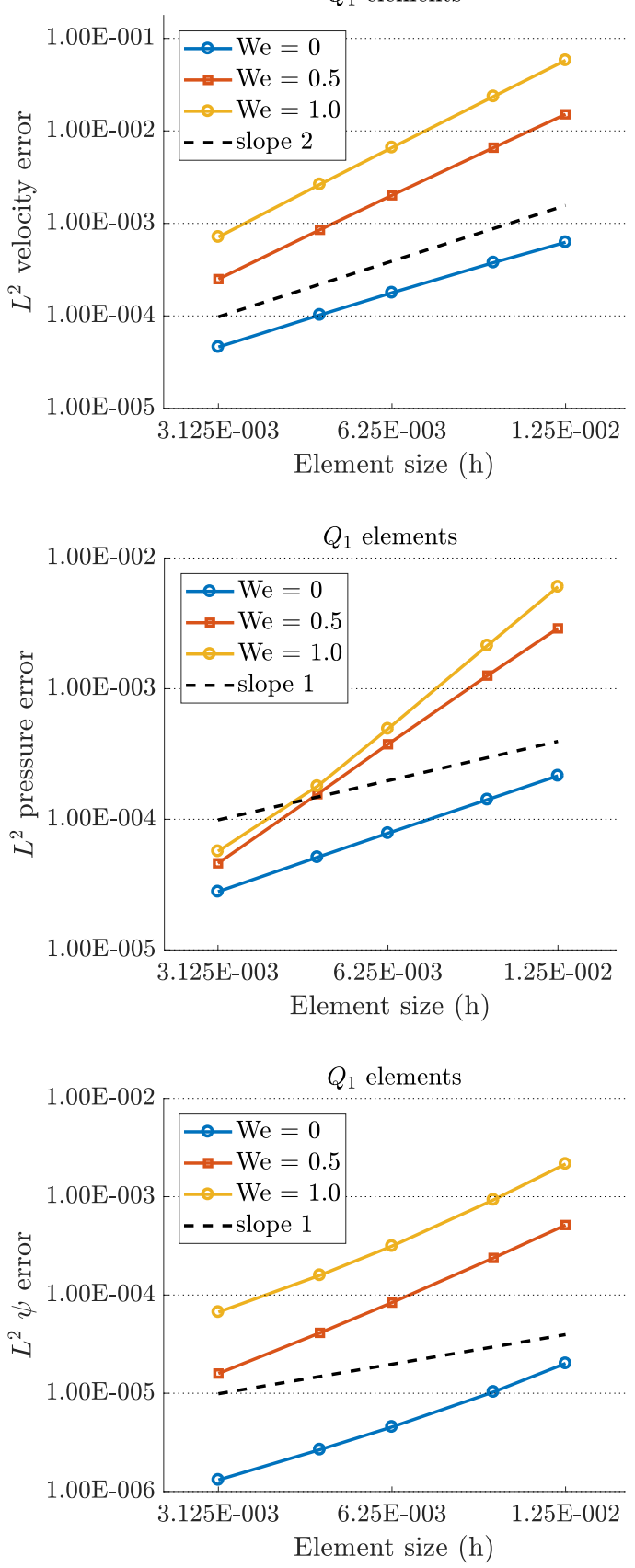

$Q_{2}$ elements
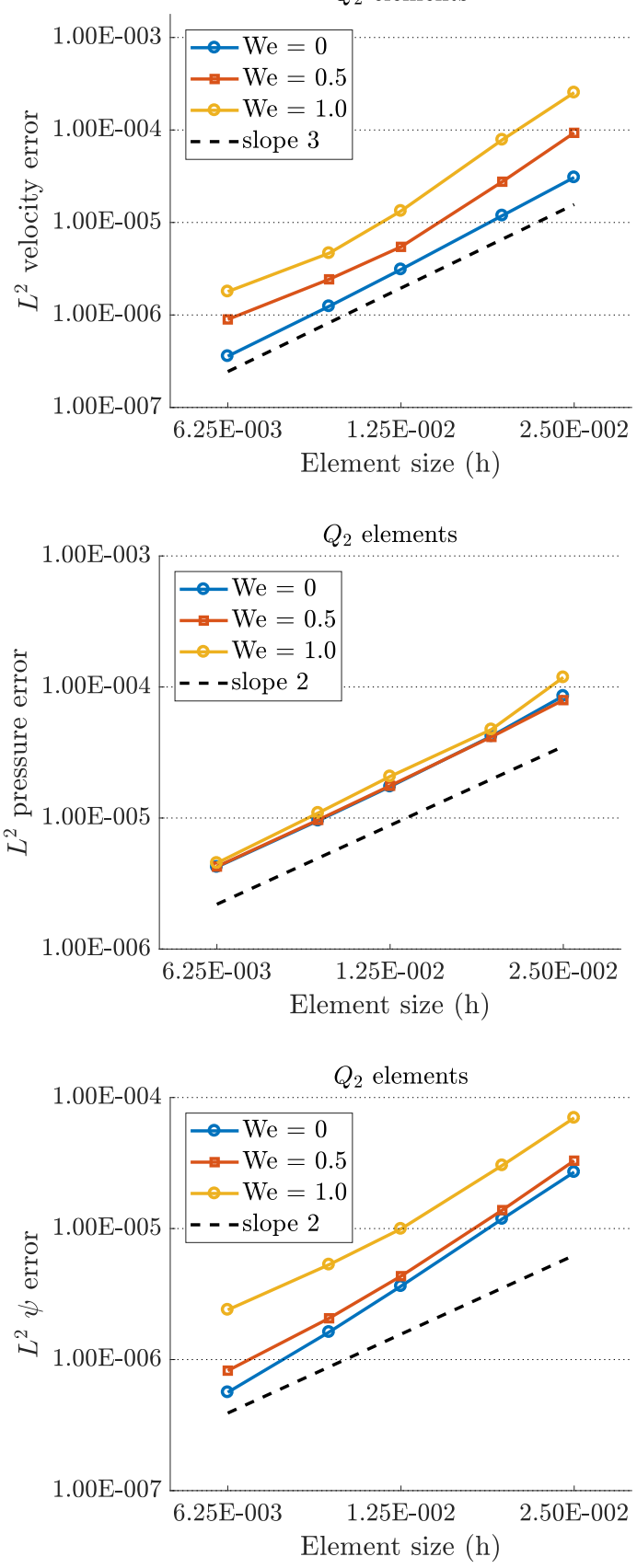

Fig. 1: Discrete $L^{2}$-errors for the manufactured exact solution.

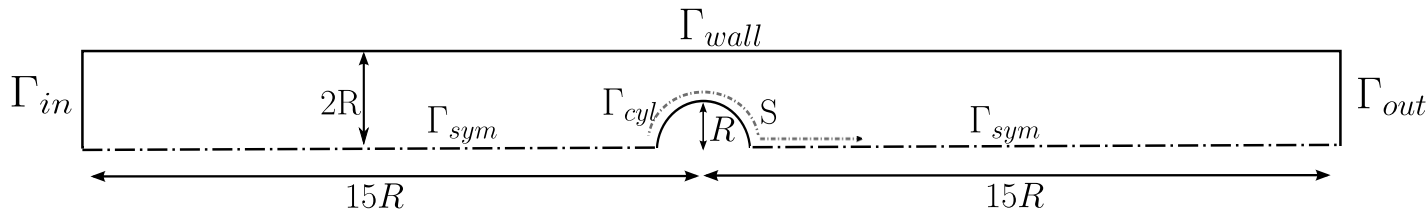

Fig. 2: Geometry and computational boundaries of the cylinder between two plates. 


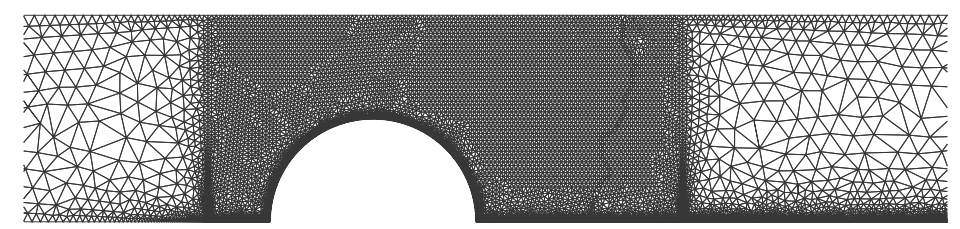

Fig. 3: Computational mesh used in flow past a cylinder.

\begin{tabular}{|c|c|c|c|}
\hline Mesh & Nodes & Elements & $h_{\min }$ \\
\hline \hline M1 & 38469 & 71980 & 0.005 \\
\hline M2 & 84378 & 156959 & 0.002 \\
\hline M3 & 179645 & 336106 & 0.001 \\
\hline
\end{tabular}

Tab. 1: Main characteristics of the computational meshes.

Let us describe the boundary conditions of the problem, following the notation included in the sketch of the geometry. For the velocity, we will impose non-slip conditions on the top wall $y=2 R, \Gamma_{\text {wall }}$, and on the cylinder boundary $\Gamma_{\text {cyl }}$, while symmetry conditions are prescribed along the axis $y=0$, denoted by $\Gamma_{\text {sym }}$. In our case, these last conditions are applied imposing the component $y$ of the velocity to be zero. On the other hand, a fully developed parabolic velocity profile and the associated elastic stress profile are imposed at the inlet $\Gamma_{\text {in }}$ :

$$
\begin{array}{cc}
u_{x}=\frac{3 Q}{2 R}\left(1-\frac{y^{2}}{(2 R)^{2}}\right), & u_{y}=0, \\
\sigma_{x x}=2 \lambda(1-\beta) \eta_{0}\left(\frac{\partial u_{x}}{\partial y}\right)^{2}, & \sigma_{x y}=(1-\beta) \eta_{0}\left(\frac{\partial u_{x}}{\partial y}\right), \quad \sigma_{y y}=0,
\end{array}
$$

where $Q$ is the flow rate and $R$ is the radius of the cylinder. Note that the stress conditions will be defined over the inflow boundary using the new variable $\boldsymbol{\psi}$, taking into account the already mentioned relation $\boldsymbol{\psi}=\log \left(\frac{\lambda_{0} \sigma}{\eta_{0}(1-\beta)}\right)$ in Section 2.2. Moreover, the horizontal velocity is left free, the vertical one is set to zero and the pressure is prescribed to zero on the outlet boundary $\Gamma_{\text {out }}$. The values for the parameters used along all this study are $Q=1, \eta_{0}=1, \beta=0.59$.

We have solved the benchmark for different Weissenberg numbers We $=\frac{\lambda U}{L_{0}}$, where the characteristic velocity in this problem is $U=\frac{3 Q}{2 R}$, and the characteristic length is $L_{0}=R$. Furthermore, in all our calculations the convective term of the momentum equation is neglected, as it is customary of this problem.

In order to check the independence of the mesh in the results we have employed three different meshes, with a similar structure. In Fig. 3 one of them is displayed, where we can observe its unstructured nature, composed of linear triangles. We have to stand out the refinement of the mesh in the region around the cylinder and downstream, where the maximum values of the stress are achieved. More details about these are found in Table 1 , which contains the number of nodes, elements and the minimum element size $h_{\text {min }}$ for each mesh.

\subsubsection{Drag coefficient results}

Although convergence for the dimensionless drag coefficient is a good indicator to check a method, in the literature some discrepancies exist when a moderately high Weissenberg number is considered (We $\geq 0.7$ ). Moreover, as the drag coefficient is an integrated quantity over a cylinder, accuracy in the whole of the domain cannot be ensured if this drag coefficient is not adequately reproduced. 


\begin{tabular}{lcccc}
\hline We & Hulsen M4 [14] & Afonso M30 [20] & Damanik [17] & P.S. \\
\hline \hline $\mathbf{0 . 1}$ & 130.363 & - & 130.366 & 130.30 \\
\hline $\mathbf{0 . 5}$ & 118.936 & 118.781 & 118.828 & 118.82 \\
\hline $\mathbf{1 . 0}$ & 118.501 & 118.662 & 118.574 & 118.88 \\
\hline $\mathbf{1 . 2}$ & 120.650 & 120.985 & 120.919 & 121.14 \\
\hline $\mathbf{1 . 4}$ & 123.587 & 124.124 & 123.936 & 124.14 \\
\hline $\mathbf{1 . 6}$ & 127.172 & 127.759 & 127.523 & 127.66 \\
\hline $\mathbf{1 . 8}$ & 131.285 & 132.024 & 131.578 & 131.53 \\
\hline $\mathbf{2 . 0}$ & 135.839 & 136.580 & 136.039 & 135.53 \\
\hline $\mathbf{2 . 2}$ & not solved & 141.801 & not solved & 139.62 \\
\hline $\mathbf{2 . 4}$ & not solved & 146.730 & not solved & 143.66 \\
\hline
\end{tabular}

Tab. 2: Comparison of drag force coefficient (P.S.: present study).

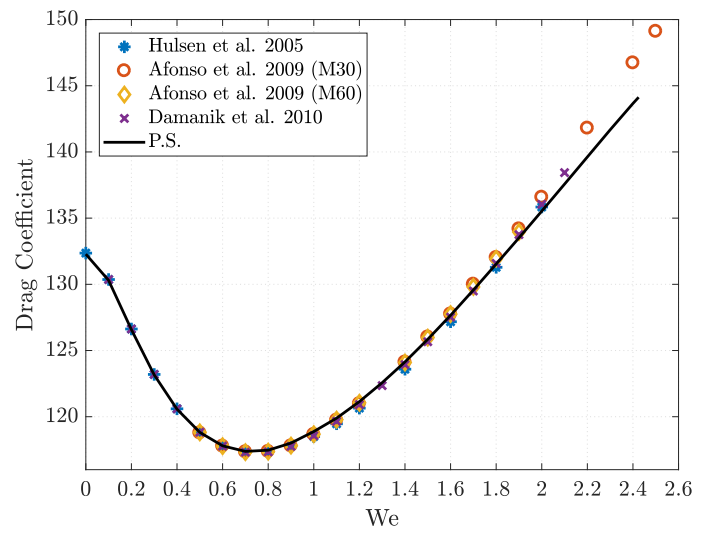

Fig. 4: Comparison of drag force coefficient (P.S.: present study).

Some drag coefficients for different Weissenberg numbers are presented numerically in Table 2 and graphically in Fig. 4, where our own results (labelled P.S.) are compared to those of other authors, who also have applied the log-conformation reformulation, such as Hulsen et al. [14], Afonso et al. [20] or Damanik et al. [17]. Particularly, Hulsen et al. [14] indicate that from a certain Weissenberg value, the solution shows time-dependency and computations do not break down, although some fluctuations are detected when $\mathrm{We}=2.0$ is reached. However, in our calculations, fluctuations have not been detected and a steady flow has been obtained.

The agreement is acceptable with other publications where the logarithmic reformulation has been made, although from values higher than 1.2 slight discrepancies are detected.

The advantages of using this formulation are notorious: while computations reported in the literature using the standard formulation break down around a Weissenberg number of 0.9 , the logarithmic formulation shows good stability for higher values. In our case, we have been capable of simulating fluids with Weissenberg number equal to 2.4. 

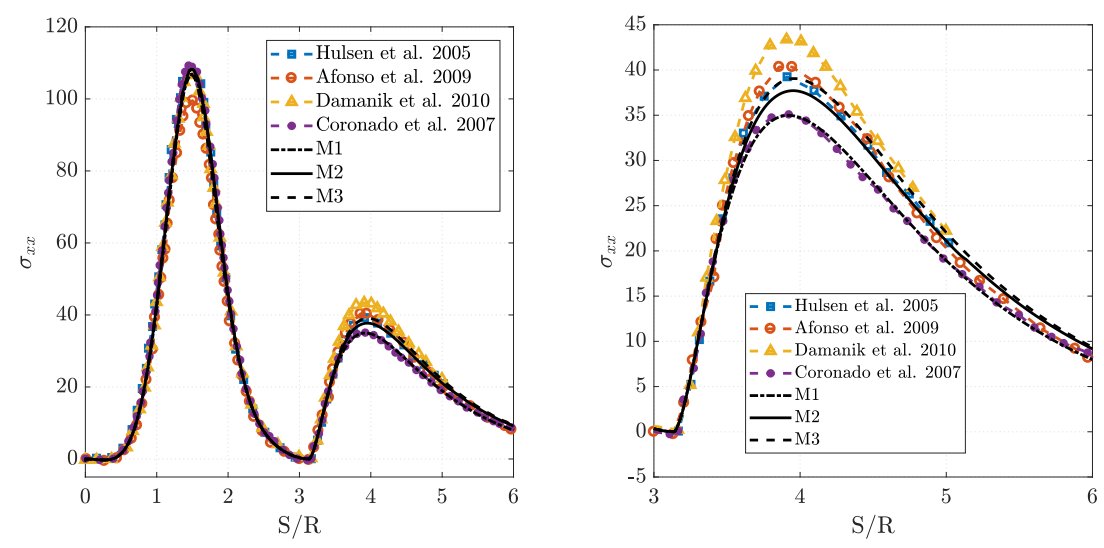

Fig. 5: Profile of the first component stress $\left(\sigma_{x x}\right)$ along cylinder and downstream for We $=$ 0.7 .

\subsubsection{Stress convergence}

As we have commented above, the drag coefficient study is not enough to prove the accuracy of the formulation. Therefore, we have displayed the component $x$ of the stress on the cylinder wall and along the downstream center line with the purpose of contrasting our results with those reported by different authors.

Regarding stress convergence when the mesh is refined, as some authors have remarked (Afonso et al. [20], Coronado et al.[15]), there are a lot of discrepancies, particularly when the Weissenberg number begins to increase (We $\geq 0.7$ ). It occurs especially for the maximum peak of stresses situated in the rear wake. Some authors, in spite of using extremely refined meshes and high order methods, cannot be conclusive about the converged results.

A comparison of the first component of the elastic stress profile along the cylinder and downstream at the center line of the domain is shown in Figures 5 and 6; our results are compared with other published results.

For Weissenberg number equal to 0.6, we are in agreement with results found in the literature, particularly with Hulsen et al. [14] and Damanik et al. [17] (not shown). However, for the figure presented associated with a Weissenberg number equal to 0.7 (Fig. 5), the discrepancies among authors start mainly in the rear wake, in spite of mesh convergence. As it has been commented previously, although this case has been computed by a wide number of authors, high order methods are not conclusive. Regarding our results, these are in agreement with the values reached in the rear wake by Hulsen et al. [14] and Afonso et al. [20] when the finest mesh is employed, although the maximum peak over the cylinder is higher than that plotted by Afonso et al. [20]; in turn, these maximum values are very close to those of the rest of authors compared. Mesh dependency needs to be highlighted for this Weissenberg number at this point, and it is shown in Fig. 5 (right), where we can see differences between meshes, especially along the centerline in the wake of the cylinder.

In Fig. 6 the results for Weissenberg 0.9 are displayed. As we have remarked before, this solution does not have mesh convergence; this phenomenon has already been reported in the literature by some researchers $[15,14,20]$. To show this effect clearly, we have plotted in Fig. 6 the values obtained with two different meshes (M1 and M2), so the lack of convergence is demonstrated, because whenever the mesh is refined the maximum values reached along the centerline downstream show a significant increment. The authors referenced above relate this to the behavior of the constitutive model, which models an unlimited extension of the fluid at finite extension rates. For this reason, numerous discrepancies are found between works published when the stress values along the rear wake 

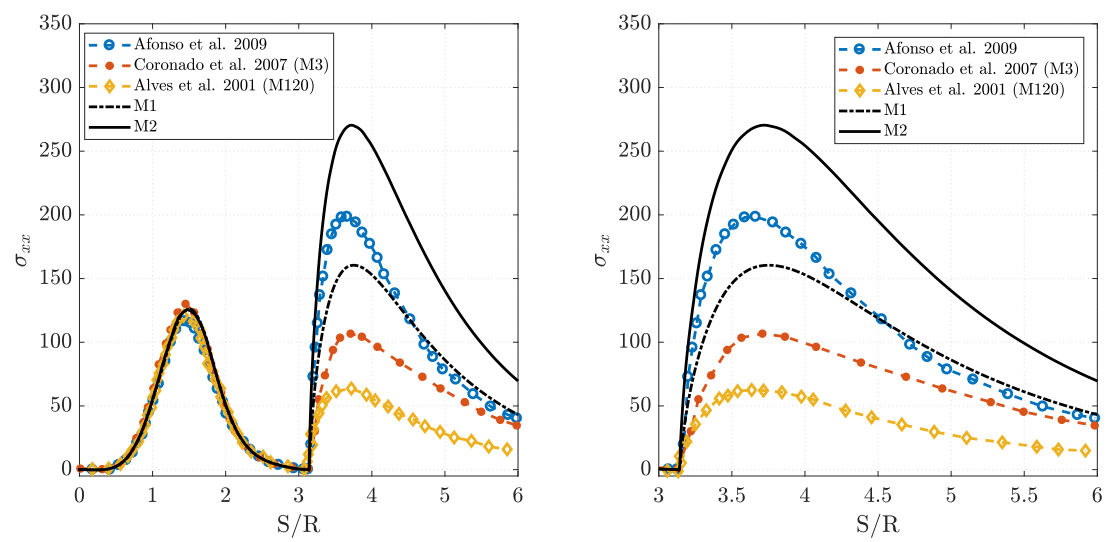

Fig. 6: Profile of the first component stress $\left(\sigma_{x x}\right)$ along cylinder and downstream for We $=$ 0.9 .

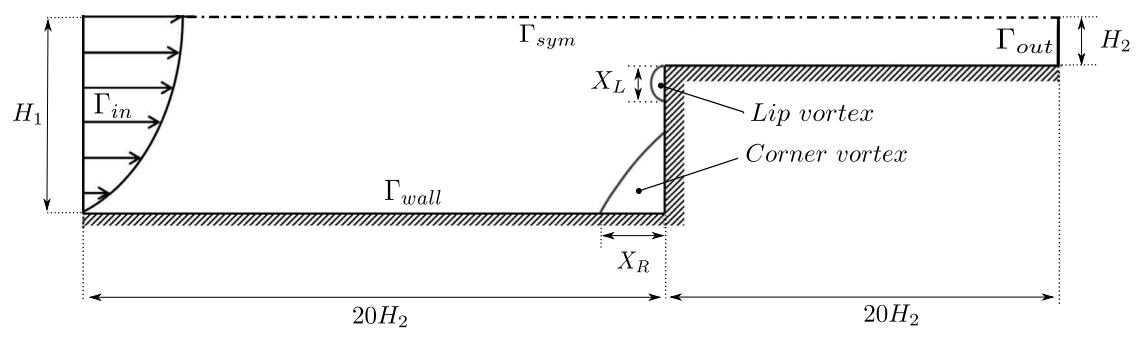

Fig. 7: Geometry of the planar contraction 4:1.

are plotted, for example between Alves et al. [39], Coronado et al.[15], Afonso et al. [20], and our own results. Nevertheless, the values in points situated around the cylinder are very close in all quoted references.

\subsection{Contraction $4: 1$}

The next benchmark presented is the well-known 4:1 planar contraction problem in the two dimensional version. This is a suitable example because it is more stringent than other benchmarks when highly elastic problems are studied, due to the singularity which is situated in the corner.

\subsubsection{Set up}

First of all, we describe the main characteristics of this case. Since the problem is symmetric, we have just considered half of the domain, as it is shown in Fig. 7. Moreover, the main characteristic lengths have been already detailed in the figure, taking $H_{1}=4$ and $H_{2}=1$, together with the corner vortex length definition denoted by $X_{R}$ and the lip vortex $X_{L}$. These quantities are useful to compare our results with those of other authors.

Let us describe the boundary conditions associated with this problem. On the solid walls $\Gamma_{\text {wall }}$, non-slip conditions are imposed for the velocity field and on symmetric boundaries $\Gamma_{\text {sym }}$, the component $y$ of the velocity is set to zero. Moreover, on the inlet boundary $\Gamma_{\text {in }}$ a fully parabolic velocity profile and stress profile are prescribed:

$$
\begin{gathered}
u_{x}=\frac{3 Q}{2 H_{1}}\left(1-\frac{y^{2}}{H_{1}^{2}}\right), \\
u_{y}=0, \\
\sigma_{x x}=2 \lambda(1-\beta) \eta_{0}\left(\frac{3 Q}{H_{1}^{3}} y\right)^{2}, \quad \sigma_{x y}=-(1-\beta) \eta_{0}\left(\frac{3 Q}{H_{1}^{3}} y\right), \quad \sigma_{y y}=0,
\end{gathered}
$$




\begin{tabular}{|c|c|c|c|}
\hline Mesh & Nodes & Elements & $\Delta x_{\min }=\Delta y_{\min }$ \\
\hline \hline M1 & 10316 & 19770 & 0.04 \\
\hline M2 & 12880 & 24712 & 0.02 \\
\hline M3 & 20441 & 39242 & 0.01 \\
\hline M4 & 28713 & 55693 & 0.0075 \\
\hline M5 & 36513 & 70818 & 0.005 \\
\hline
\end{tabular}

Tab. 3: Characteristic of the meshes of the 4:1 contraction problem.

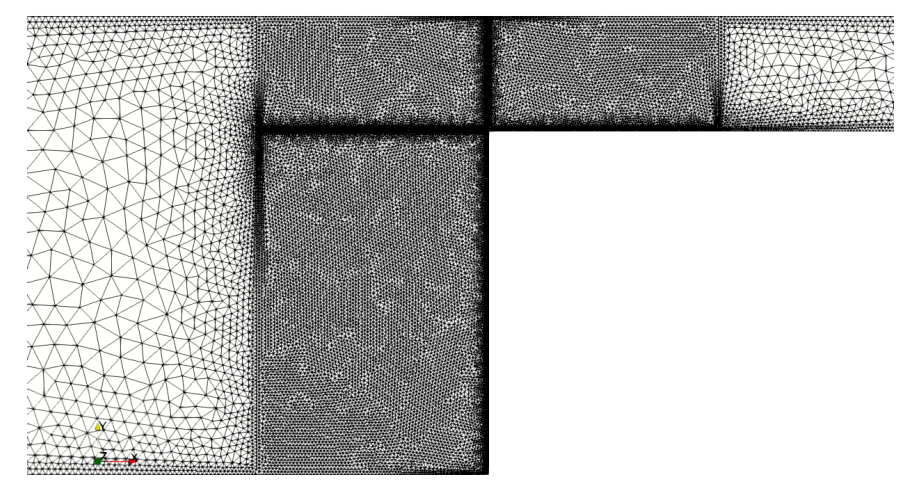

Fig. 8: Mesh used in contraction 4:1 problem.

where $Q$ is the flow rate, set to 1 . In this case the characteristic length is $H_{2}=1$, which is the length of the inlet channel, and the characteristic velocity is the mean outflow velocity, $\bar{u}_{2}=1$. Note that the stress conditions will be imposed to the new variable $\boldsymbol{\psi}$, easily computed from the defined stress functions; these are required in order to avoid the need of using a too large computational domain.

For the outlet boundary $\Gamma_{\text {out }}$, the $x$-component of the velocity is left free, and the $y$-component is set to zero. In addition, the $x$-component of the normal component of the total Cauchy stress tensor is set to zero. The remaining parameters are $\eta_{0}=1$ and $\beta=1 / 9$.

With the characteristic values chosen, We $=\frac{\lambda \bar{u}_{2}}{H_{2}}$ and the Reynolds number is

$$
\operatorname{Re}=\frac{\rho \bar{u}_{2} H_{2}}{\eta_{0}},
$$

where parameter $\rho$ is the fluid's density.

The main characteristics of the different size meshes used are detailed in Table 3 . The structure is shared by all of them, although only one is shown in Fig. 8. The notation $\Delta x_{\min }=\Delta y_{\min }$ indicates the minimum element sizes in the $x$ and $y$ directions. We have to remark the structured character of the mesh employed near to the contraction, while it is unstructured in the rest of the domain. The results displayed in Section 4.3.2 correspond to mesh M3, while in Section 4.3.3 various mesh sizes are used.

\subsubsection{Oldroyd-B flow at $\operatorname{Re}=1$}

In this subsection we will study the problem taking into consideration the inertial effects, in other words, without neglecting the convective term of the momentum equation. So, in order to be capable of comparing our results with others, we have chosen the Reynolds number to be equal to 1 . All the authors found in the literature have solved this exact 
case employing the standard formulation. A wide range of techniques have been carried out to solve this problem: Sato and Richardson [42] and Phillips and Williams et al. [43] describe a semi-Lagrangian finite volume scheme, Nithiarasu et al. [44] propose an explicit characteristic based split (CBS) scheme, whereas Li et al [45] present a mixed finite element scheme, utilizing the DEVSS method for stress stabilization. Castillo et al. [2] proposed a stabilized method using the VMS method with a discontinuity capturing technique which allows to deal with local discontinuities.

In our case, the maximum Weissenberg number achieved by the present scheme is about We $=9.0$. This value is smaller than the Weissenberg value that we have been capable of simulating when the Reynolds number is set to zero. This effect is in agreement with the authors quoted before, and is produced by the relevance of the non-linear convective term of the momentum equation. As it is commented before, we have not come upon this problem solved with the logarithmic reformulation in the literature, but the benefits of this formulation are clear in comparison with the standard formulation, allowing us to solve the problem for fluids with much higher elasticity. For example, one of the highest values reached for the standard formulation is $\mathrm{We}=5.0$ in the work written by Castillo et al. [2], where it is explained that for $\mathrm{We}=5.5$ some instabilities appeared, and the scheme is incapable of solving the problem for $\mathrm{We}=6.0$.

Next, we will present some results, starting with the distribution of streamlines near the contraction for some of Weissenberg numbers, shown in Fig. 9. As in other works, the vortex in the corner decreases when We increases, while the lip vortex grows progressively for increasing Weissenberg numbers. This secondary vortex starts to emerge for $\mathrm{We} \geq 1.0$ (see $[44,45,2]$ for discussion). Lip vortices start to merge with the corner vortices at $\mathrm{We}=5.5$ approximately, but two different centers of rotation are clearly defined. The two centers merge at about $\mathrm{We}=6.0$. The resulting corner, in some works as [23], is referred as the third vortex. So, when the Weissenberg number increases, we observe a divergence of the streamlines upstream of the contraction.

The relationship between the corner vortex length (denoted by $X_{R}$ in Fig. 7) and the Weissenberg number is shown in in Fig. 10 together with the results of other works in the literature. We have to remark that although the corner vortex sizes are consistent with results published, lip vortex sizes are notably higher than the ones reported in the literature. However, it seems a logical behavior that contributes to the final merge between the two vortices. Both graphs are plotted up to Weissenberg 5.0 because above this value the third vortex appears, annihilating the previous vortices. This phenomenon is clearly visible in Fig. 9.

The first elastic stress distribution $\left(\sigma_{x x}\right)$ along $y=-H_{2}$ is given in Fig. 11 for various Weissenberg values using mesh M3. The singularity (at the corner) is located at $x=20.0$. We can observe that the peak values of the viscoelastic stresses increase with elasticity. We have found differences between the maximum peaks obtained and the results presented in [2], where the standard formulation is employed. Nevertheless, our solution is very close to the distribution shown in the work of Afonso et al. [21], where a study comparing both formulation is developed and where a distribution of the elastic stress along the centerline $y=0$ (symmetry axis), where the solution is smoother, is plotted. By following the same idea, Fig. 12 compares solutions in both formulations; we have not found large discrepancies between them, except in the maximum values, where a slight variation is observed when elasticity grows. Moreover, in [21] it is described how these differences are more perceptible from $\mathrm{We}=2.5$, because with the logarithmic formulation the flow becomes unsteady, while with the standard one it diverges at $\mathrm{We}=3.0$. 


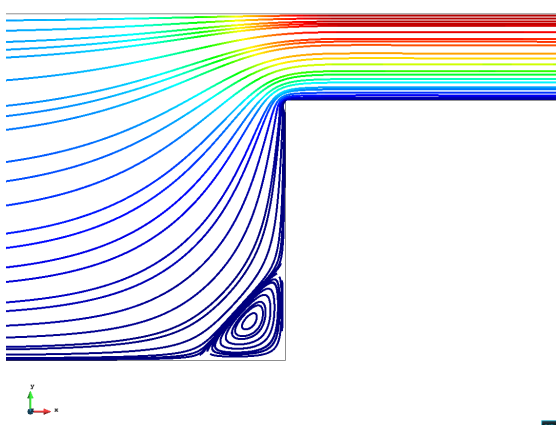

(a) $\mathrm{We}=1.0$

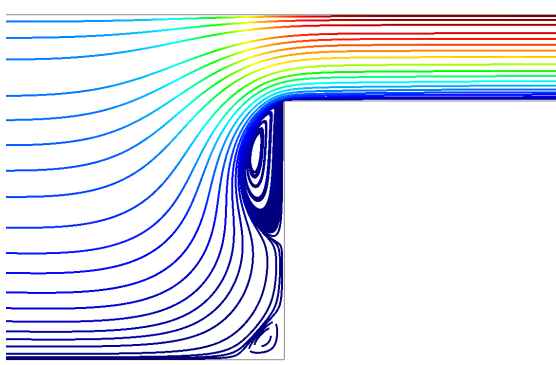

L.

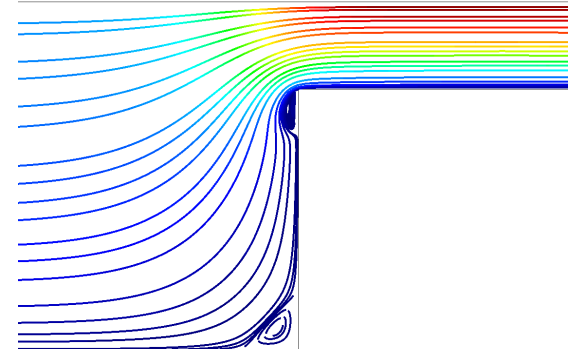

(b) $\mathrm{We}=3.0$

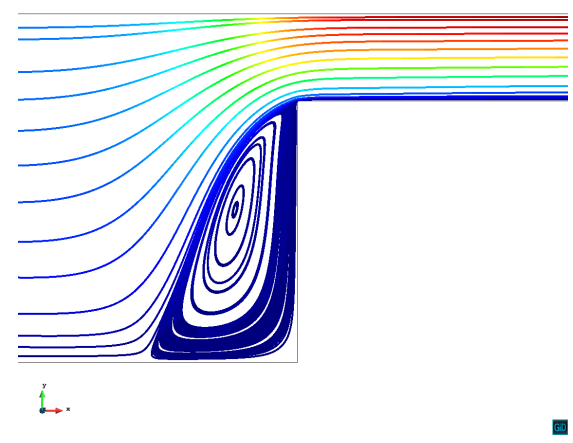

(d) $\mathrm{We}=7.0$

(c) $\mathrm{We}=5.0$

Fig. 9: Streamlines patterns in the contraction planar for different Weissenberg number for $\operatorname{Re}=1.0$.

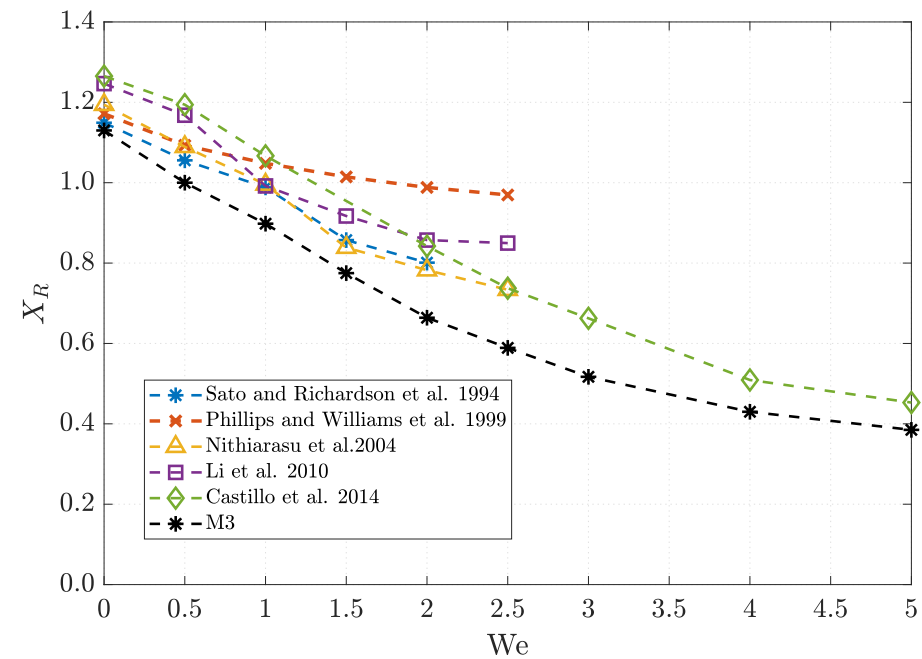

Fig. 10: Corner length comparison for $\mathrm{Re}=1.0, \mathrm{We} \leq 5.0$. 


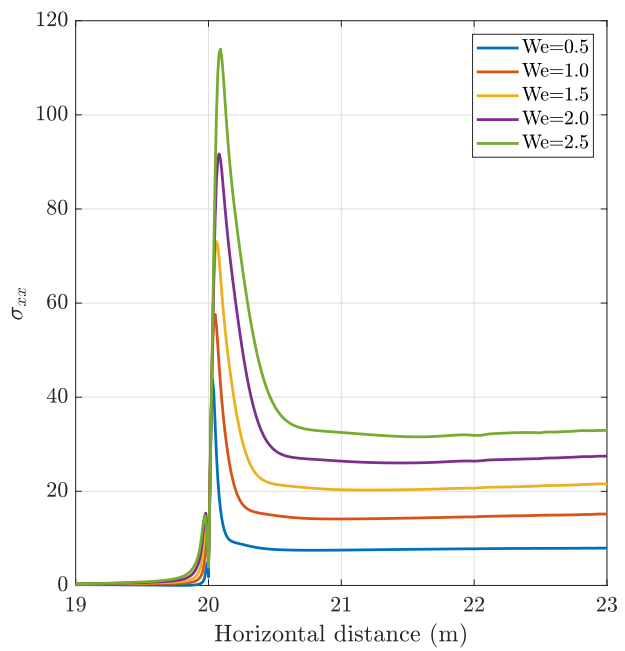

Fig. 11: First elastic stress component along $y=-H_{2}, \operatorname{Re}=1.0$.

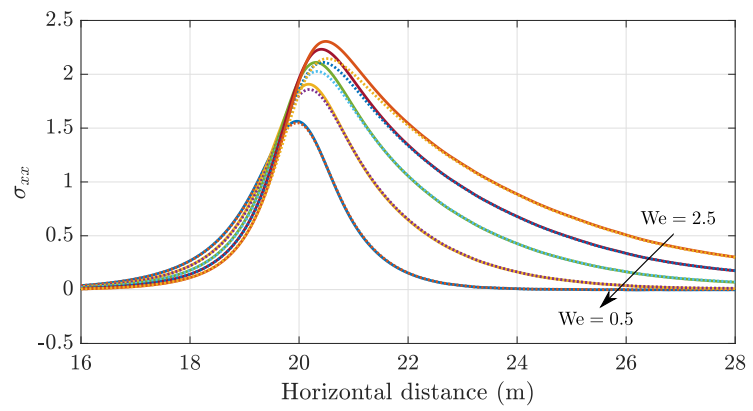

Fig. 12: First elastic stress component along $y=0, \operatorname{Re}=1.0$. 

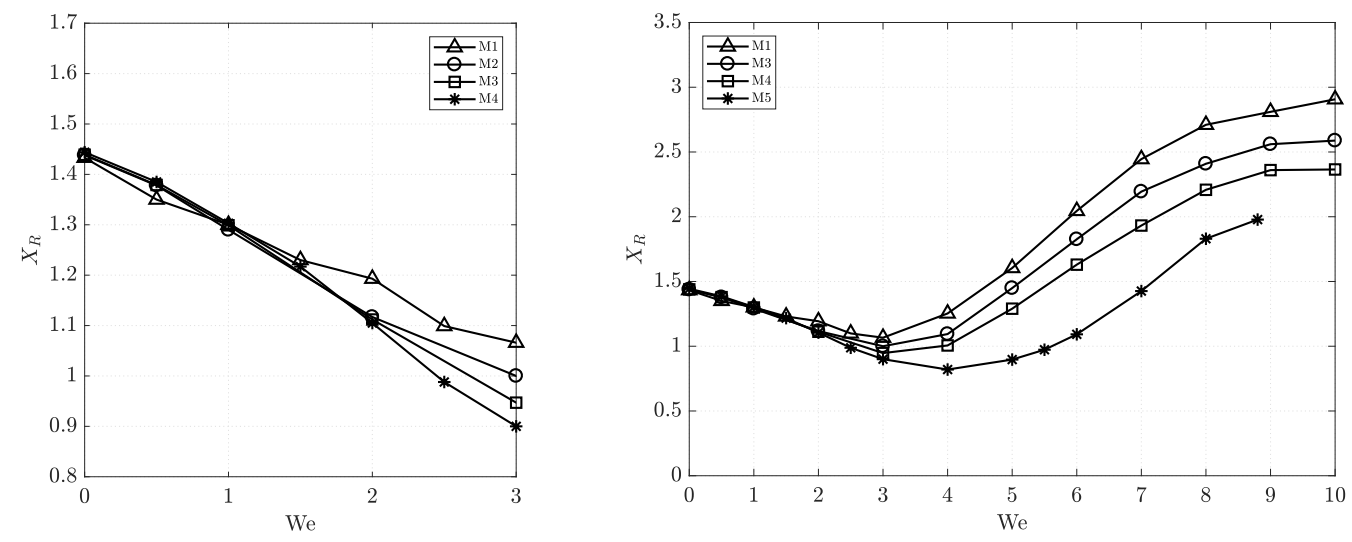

Fig. 13: Corner vortex length comparison between meshes for $\mathrm{Re}=0.01$.

\subsubsection{Oldroyd-B flow at $\operatorname{Re}=0.01$}

There are studies, such as Sato and Richardson [42] and Matallah et al. [46], which indicate that solutions for the Oldroyd-B flow with a Reynolds number Re $\leq 0.01$ are almost identical to those for the Oldroyd-B flow with $\mathrm{Re}=0.0$, in other words, those of creeping flow. For this reason, the solutions for $\mathrm{Re}=0.01$ given by the proposed scheme can be compared with studies of creeping flow published in the literature. Recall that in our case Re $=\frac{\rho \bar{\alpha}_{2} H_{2}}{\eta_{0}}$.

Many references with different numerical schemes present results for the problem considered. For example, Alves et al. [47], analyze the dispersion and the vortex length using several methods, and Kim et al. [48] employ a transient finite element method based on a fractional step scheme and stabilization techniques. Authors quoted in previous sections, as $[44,2]$, also validate their schemes for $R e=0.01$; these are included in our comparisons. All the mentioned articles use the standard formulation; however, we have come upon some relevant analysis where a log-conformation reformulation is employed to solve the contraction problem, such as the work by Afonso et al. [21], where a dynamic evaluation of the behavior and the fluctuation of flow at high Weissenberg number is studied, Comminal et al. [23], which presents a numerical solution for a Weissenberg number up to 20 with a streamfunction-log-conformation methodology, and Pimenta et al. [25], the most recent study, in which the typical solver available in the OpenFOAM toolbox is modified to get second-order accuracy. All these works consider We $\leq 12$.

The maximum Weissenberg number reached in our case is 15.0 in stationary regime and using the coarsest mesh. We are aware of the lack of accuracy of the results of the highest we reached, because in references [21, 23] the existence of large fluctuations are described for high Weissenberg numbers, whereas we obtain a stable stationary solution. Note that the aim of this work is to validate the formulation, therefore transient terms have just been added to achieve stationary solutions and not to perform truly transient calculations.

The behavior of the vortex is very close to the one described for the $R e=1.0$ case. Fig. 13 shows the evolution of the corner vortex and later the third vortex size when the Weissenberg number is increased for the three finest meshes. Just as it is exposed in the work of Pimenta et al. [25], the growth of the lip vortex size is shown to be significantly dependent on the mesh resolution, where finer meshes lead to a smaller lip vortex and, consequently, it affects the merge between two vortices and thus to the growth and size of the third vortex.

In Fig. 14, the comparison with the literature (references [21, 23]) of the corner size in terms of the Weissenberg number is shown. A survey of the numerical results presented in 

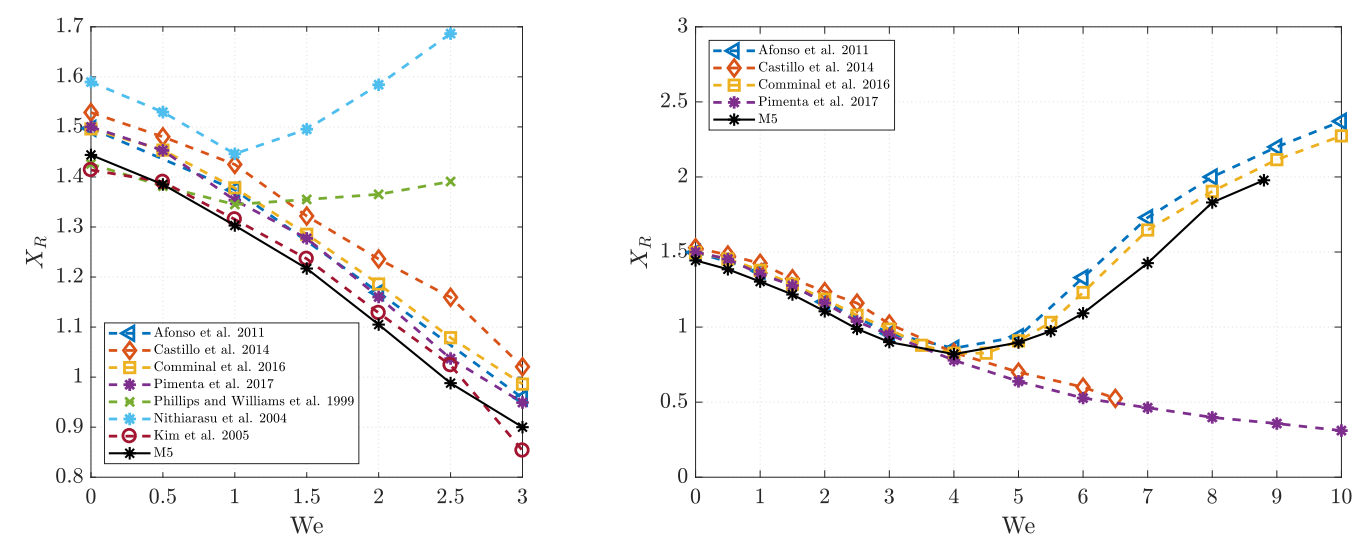

Fig. 14: Corner vortex length comparison for $\mathrm{Re}=0.01$.

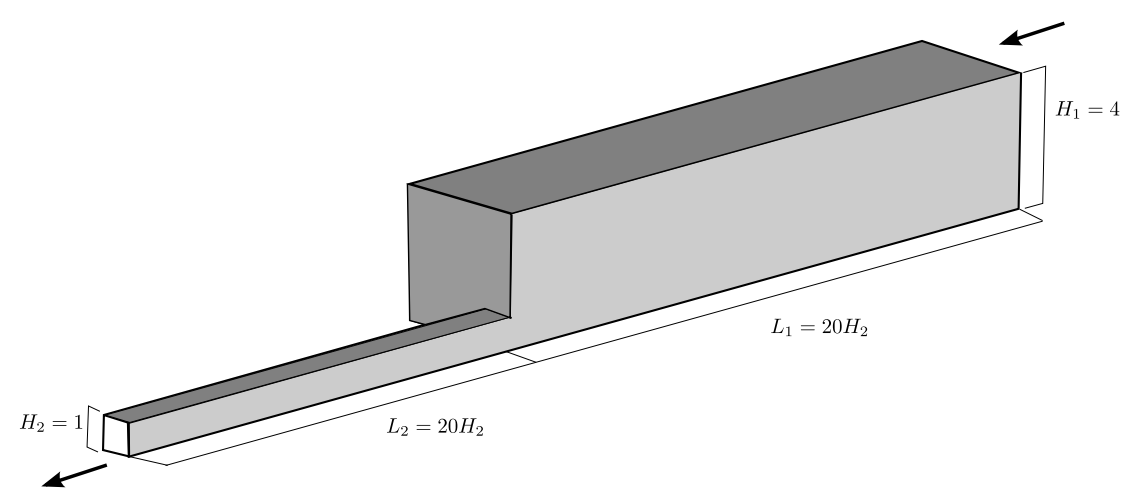

Fig. 15: Three dimensional geometry of the $4: 1$ contraction problem.

the literature reveals large discrepancies between the results of the different studies. Our results are in very good agreement with the solutions of Kim et al. [48] for values up to $\mathrm{We}=3.0$.

\subsection{Three dimensional case}

As a last example, we are going to show a 4:1 contraction problem in its three dimensional version, in order to proof that the proposed formulation is ready for 3D problems. Measures and conditions considered are similar to those in [2].

\subsubsection{Set up}

The geometry is illustrated in Fig. 15 together with some measures. Since the problem is symmetric for low Weissenberg numbers, we have just considered a fourth of the total domain. In [21], the full domain was used in order to be able to capture flow asymmetries or instabilities when the Weissenberg number grows. Both inlet and outlet lengths are the same as in the two dimensional case, long enough for getting a full flow development. Moreover, the characteristic length we have chosen is $H_{2}=1$, which is half of the channel width of the narrowest part, and the average velocity in the outlet of the channel $\bar{u}_{2}=1$ has been chosen as characteristic velocity.

The boundary conditions are an extension from the two dimensional case, but now the domain has two symmetry boundaries instead of one. The symmetry planes are $y=0$ and $z=0$, and the normal velocity to each plane is set to zero. In this problem we have just considered a Weissenberg number of $\mathrm{We}=1.0$ and the inertial effects have been minimized, taking $\operatorname{Re}=0.01$. 


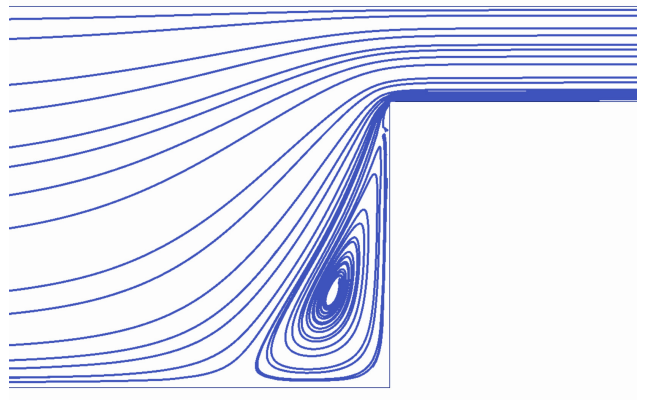

(a) Cut with the plane $y=0$.

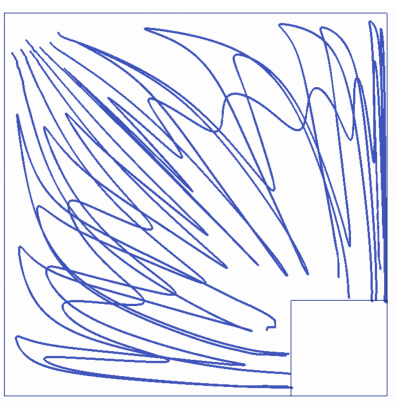

(b) Cut plane a $x=$ cte near to the contraction plane.

Fig. 16: Streamlines in the three dimensional 4:1 planar contraction in two different views.

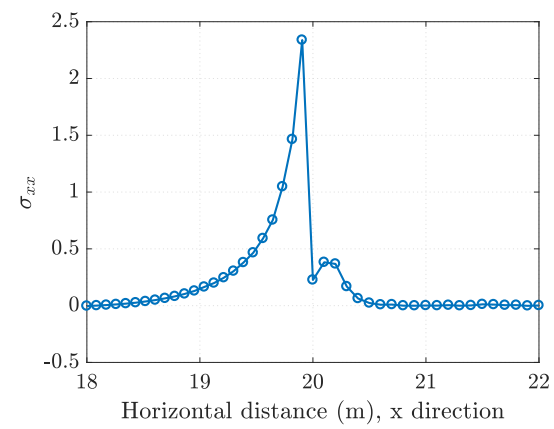

Fig. 17: Normal elastic stress component $\sigma_{x x}$ along the corner.

Higher values of the Weissenberg number would require finer meshes in order to be capable to capture the lip vortex. Nevertheless, we are now trying to study the behavior of the highest Weissenberg number in a three dimensional geometry, as it is done in [2]. The mesh used is formed by 476852 unstructured tetrahedra and 86856 nodes.

\subsubsection{Some results}

Only a few pictures of results will be presented, intending to give an idea of the flow. On the one side, streamlines are shown in Fig. 16, where these are plotted on two perpendicular planes. Particularly, in Fig. 16a the main corner vortex is clearly appreciated, and also the lip vortex starts to emerge. The similarities with the two dimensional case are remarkable, in spite of the minimum mesh size being notably finer in that problem. In Fig. 16b the symmetry with respect to plane $y=z$ of the streamlines can be observed.

Lastly, in Fig. 17 the first elastic stress component along the wall around the contraction corner is shown, and we can appreciate how the stress peak is captured.

We have to stand out that the results presented in this section pretend to make a qualitative study of the three dimensional problem, neither comparing the results obtained with other publications nor establishing a rigorous analysis of the differences between them.

\section{Conclusions}

In this work a FE method has been designed for the log-conformation formulation of Fattal and Kupferman [1], considering a modification with respect to the original formulation which is non-singular with respect to the relaxation time parameter and, moreover, that allows a direct steady numerical computation. The spatial approximation is carried out using a stabilized FE method based on the VMS framework. Firstly, a residual-based 
formulation of the equations is presented, and later the split OSS method is developed for the momentum and continuity equations, whereas the constitutive equation can be stabilized using the classical residual-based stabilization, following the steps of [2]. The linearization of the problem has been presented in detail, emphasizing the treatment of the exponential function. The convergence of the proposed method has a strong dependency on this treatment.

The resulting method allows one to obtain globally stable solutions, and has been validated in different benchmarks for high Weissenberg numbers, showing accuracy, optimal convergence for smooth solutions, and robustness even in steady-state computations, reaching accurate results in comparison with other methods reported in the literature. Moreover, the formulation also performs well in the three-dimensional case, with good results from a qualitative point of view.

\section{Acknowledgements}

L. Moreno acknowledges the support received from the Spanish Governement through a predoctoral FPI Grant. R. Codina acknowledges the support received from the ICREA Acadèmia Research Program of the Catalan Government. J. Baiges acknowledges the support of the Spanish Government through the Ramón y Cajal grant RYC-2015-17367. E Castillo acknowledges the support of the Chilean Council for Scientific and Technological Research through the project CONICYT-FONDECYT 11160160. This work is partially funded through the ELASTIC-FLOW project, Ref. DPI2015-67857-R of the Spanish Government.

\section{References}

[1] R. Fattal and R. Kupferman. Constitutive laws for the matrix-logarithm of the conformation tensor. Journal of Non-Newtonian Fluid Mechanics, 123(2-3):281-285, 2004 .

[2] E. Castillo and R. Codina. Variational multi-scale stabilized formulations for the stationary three-field incompressible viscoelastic flow problem. Computer Methods in Applied Mechanics and Engineering, 279:579-605, 2014.

[3] M. D. Graham. Drag reduction in turbulent flow of polymer solutions. Rheology reviews, 2(2):143-170, 2004.

[4] A. Groisman and V. Steinberg. Elastic turbulence in a polymer solution flow. Nature, 405(6782):53, 2000 .

[5] J.M. Dealy. Weissenberg and deborah numbers-their definition and use. Rheol. Bull, 79(2):14-18, 2010.

[6] R. G. Owens and T. N. Phillips. Computational rheology, volume 14. World Scientific, 2002 .

[7] R. Fattal and R. Kupferman. Time-dependent simulation of viscoelastic flows at high weissenberg number using the log-conformation representation. Journal of NonNewtonian Fluid Mechanics, 126(1):23-37, 2005.

[8] A.I. Leonov. Analysis of simple constitutive equations for viscoelastic liquids. Journal of non-Newtonian fluid mechanics, 42(3):323-350, 1992.

[9] Y. Kwon. Recent results on the analysis of viscoelastic constitutive equations. KoreaAustralia Rheology Journal, 14(1):33-45, 2002. 
[10] M.A. Hulsen, A.P.G. Van Heel, and B.H.A.A. Van Den Brule. Simulation of viscoelastic flows using Brownian configuration fields. Journal of Non-Newtonian Fluid Mechanics, 70(1-2):79-101, 1997.

[11] T. Vaithianathan and L. R. Collins. Numerical approach to simulating turbulent flow of a viscoelastic polymer solution. Journal of Computational Physics, 187(1):1-21, 2003.

[12] N. Balci, B. Thomases, M. Renardy, and C. R. Doering. Symmetric factorization of the conformation tensor in viscoelastic fluid models. Journal of Non-Newtonian Fluid Mechanics, 166(11):546-553, 2011.

[13] A.M. Afonso, F.T. Pinho, and M.A. Alves. The kernel-conformation constitutive laws. Journal of Non-Newtonian Fluid Mechanics, 167:30-37, 2012.

[14] M. A Hulsen, R. Fattal, and R. Kupferman. Flow of viscoelastic fluids past a cylinder at high weissenberg number: stabilized simulations using matrix logarithms. Journal of Non-Newtonian Fluid Mechanics, 127(1):27-39, 2005.

[15] O. M. Coronado, D. Arora, M. Behr, and M. Pasquali. A simple method for simulating general viscoelastic fluid flows with an alternate log-conformation formulation. Journal of Non-Newtonian Fluid Mechanics, 147(3):189-199, 2007.

[16] A. Kane, R. Guénette, and A. Fortin. A comparison of four implementations of the log-conformation formulation for viscoelastic fluid flows. Journal of Non-Newtonian Fluid Mechanics, 164(1-3):45-50, 2009.

[17] H. Damanik, J. Hron, A. Ouazzi, and S. Turek. A monolithic fem approach for the log-conformation reformulation (lcr) of viscoelastic flow problems. Journal of NonNewtonian Fluid Mechanics, 165(19-20):1105-1113, 2010.

[18] P. Saramito. On a modified non-singular log-conformation formulation for johnsonsegalman viscoelastic fluids. Journal of Non-Newtonian Fluid Mechanics, 211:16-30, 2014 .

[19] P. Knechtges, M. Behr, and S. Elgeti. Fully-implicit log-conformation formulation of constitutive laws. Journal of Non-Newtonian Fluid Mechanics, 214:78-87, 2014.

[20] A.M. Afonso, P.J. Oliveira, F. T. de Pinho, and M.A. Alves. The log-conformation tensor approach in the finite-volume method framework. Journal of Non-Newtonian Fluid Mechanics, 157(1-2):55-65, 2009.

[21] A.M. Afonso, P.J. Oliveira, F.T. Pinho, and M.A. Alves. Dynamics of high-deborahnumber entry flows: a numerical study. Journal of Fluid Mechanics, 677:272-304, 2011.

[22] R. Comminal, J. Spangenberg, and J. H. Hattel. Robust simulations of viscoelastic flows at high weissenberg numbers with the streamfunction/log-conformation formulation. Journal of Non-Newtonian Fluid Mechanics, 223:37-61, 2015.

[23] R. Comminal, J. H. Hattel, M. A. Alves, and J. Spangenberg. Vortex behavior of the oldroyd-b fluid in the 4-1 planar contraction simulated with the streamfunctionlog-conformation formulation. Journal of Non-Newtonian Fluid Mechanics, 237:1-15, 2016. 
[24] R. Kupferman. A central-difference scheme for a pure stream function formulation of incompressible viscous flow. SIAM Journal on Scientific Computing, 23(1):1-18, 2001.

[25] F. Pimenta and M.A. Alves. Stabilization of an open-source finite-volume solver for viscoelastic fluid flows. Journal of Non-Newtonian Fluid Mechanics, 239:85-104, 2017.

[26] T.J.R. Hughes, G. R. Feijóo, L. Mazzei, and J. Quincy. The variational multiscale method. a paradigm for computational mechanics. Computer methods in applied mechanics and engineering, 166(1-2):3-24, 1998.

[27] R. Codina. Stabilization of incompressibility and convection through orthogonal subscales in finite element methods. Computer methods in applied mechanics and engineering, 190(13-14):1579-1599, 2000.

[28] R. Codina. Stabilized finite element approximation of transient incompressible flows using orthogonal subscales. Computer Methods in Applied Mechanics and Engineering, 191:4295-4321, 2002.

[29] R. Codina. Analysis of a stabilized finite element approximation of the Oseen equations using orthogonal subscales. Applied Numerical Mathematics, 58:264-283, 2008.

[30] E. Castillo and R. Codina. Stabilized stress-velocity-pressure finite element formulations of the Navier-Stokes problem for fluids with non-linear viscosity. Computer methods in applied mechanics and engineering, 279:554-578, 2014.

[31] E. Castillo and R. Codina. Numerical analysis of a stabilized finite element approximation for the three-field linearized viscoelastic fluid problem using arbitrary interpolations. ESAIM: Mathematical Modelling and Numerical Analysis, 51(4):1407-1427, 2017.

[32] G. R. Barrenechea, E. Castillo, and R. Codina. Time-dependent semidiscrete analysis of the viscoelastic fluid flow problem using a variational multiscale stabilized formulation. IMA Journal of Numerical Analysis, 2018.

[33] J. Kwack and A. Masud. A three-field formulation for incompressible viscoelastic fluids. International Journal of Engineering Science, 48(11):1413-1432, 2010.

[34] J. Kwack, A. Masud, and K.R. Rajagopal. Stabilized mixed three-field formulation for a generalized incompressible oldroyd-b model. International Journal for Numerical Methods in Fluids, 83(9):704-734, 2017.

[35] E. Castillo and R. Codina. First, second and third order fractional step methods for the three-field viscoelastic flow problem. Journal of Computational Physics, 296:113$137,2015$.

[36] R. Codina. Finite element approximation of the three-field formulation of the stokes problem using arbitrary interpolations. SIAM Journal on Numerical Analysis, 47(1):699-718, 2009.

[37] E. Castillo and R. Codina. Finite element approximation of the viscoelastic flow problem: A non-residual based stabilized formulation. Computers \& Fluids, 142:7278, 2017. 
[38] Y. Fan, R.I. Tanner, and N. Phan-Thien. Galerkin/least-square finite-element methods for steady viscoelastic flows. Journal of Non-Newtonian Fluid Mechanics, 84(23):233-256, 1999.

[39] M.A. Alves, F.T. Pinho, and P.J. Oliveira. The flow of viscoelastic fluids past a cylinder: finite-volume high-resolution methods. Journal of Non-Newtonian Fluid Mechanics, 97(2-3):207-232, 2001.

[40] A.E. Caola, Y.L. Joo, R.C. Armstrong, and R.A. Brown. Highly parallel time integration of viscoelastic flows. Journal of non-newtonian fluid mechanics, 100(1-3):191$216,2001$.

[41] R. G. Owens, C. Chauvière, and T. N. Philips. A locally-upwinded spectral technique (lust) for viscoelastic flows. Journal of non-newtonian fluid mechanics, 108(1-3):49$71,2002$.

[42] T. Sato and S. M. Richardson. Explicit numerical simulation of time-dependent viscoelastic flow problems by a finite element/finite volume method. Journal of NonNewtonian Fluid Mechanics, 51(3):249-275, 1994.

[43] T.N. Phillips and A.J. Williams. Viscoelastic flow through a planar contraction using a semi-lagrangian finite volume method. Journal of Non-Newtonian Fluid Mechanics, 87(2-3):215-246, 1999.

[44] P. Nithiarasu. A fully explicit characteristic based split (cbs) scheme for viscoelastic flow calculations. International journal for numerical methods in engineering, 60(5):949-978, 2004.

[45] X. Li, X. Han, and X. Wang. Numerical modeling of viscoelastic flows using equal low-order finite elements. Computer Methods in Applied Mechanics and Engineering, 199(9-12):570-581, 2010.

[46] H. Matallah, P. Townsend, and M.F. Webster. Recovery and stress-splitting schemes for viscoelastic flows. Journal of Non-Newtonian Fluid Mechanics, 75(2-3):139-166, 1998.

[47] M.A. Alves, F.T. Pinho, and P.J. Oliveira. Effect of a high-resolution differencing scheme on finite-volume predictions of viscoelastic flows. Journal of Non-Newtonian Fluid Mechanics, 93(2-3):287-314, 2000.

[48] J. M. Kim, C. Kim, J. H. Kim, C. Chung, K. H. Ahn, and S. Jong Lee. High-resolution finite element simulation of 4: 1 planar contraction flow of viscoelastic fluid. Journal of non-newtonian fluid mechanics, 129(1):23-37, 2005. 